\title{
An RNA-binding protein, Qki5, regulates embryonic neural stem cells through pre-mRNA processing in cell adhesion signaling
}

\author{
Yoshika Hayakawa-Yano, ${ }^{1,6}$ Satoshi Suyama, ${ }^{2,6}$ Masahiro Nogami, ${ }^{3,4}$ Masato Yugami, $^{3,4}$ Ikuko Koya, ${ }^{2}$ \\ Takako Furukawa, ${ }^{1}$ Li Zhou, ${ }^{5}$ Manabu Abe, ${ }^{5}$ Kenji Sakimura, ${ }^{5}$ Hirohide Takebayashi, ${ }^{1}$ \\ Atsushi Nakanishi, ${ }^{3,4}$ Hideyuki Okano, ${ }^{2}$ and Masato Yano ${ }^{1,2}$ \\ ${ }^{1}$ Division of Neurobiology and Anatomy, Graduate School of Medical and Dental Sciences, Niigata University, Asahimachidori, \\ Chuo-ku, Niigata, Niigata 951-8510, Japan; ${ }^{2}$ Department of Physiology, Keio University School of Medicine, Shinjuku-ku, Tokyo \\ 160-8582, Japan; ${ }^{3}$ Shonan Incubation Laboratories, Pharmaceutical Research Division, Takeda Pharmaceutical Company Limited, \\ Fujisawa, Kanagawa 251-8555, Japan; ${ }^{4}$ Integrated Technology Research Laboratories, Pharmaceutical Research Division, Takeda \\ Pharmaceutical Company Limited, Fujisawa, Kanagawa 251-8555, Japan; ${ }^{5}$ Department of Cellular Neurobiology, Brain Research \\ Institute, Niigata University, Asahimachidori, Chuo-ku, Niigata, Niigata 951-8585, Japan
}

Cell type-specific transcriptomes are enabled by the action of multiple regulators, which are frequently expressed within restricted tissue regions. In the present study, we identify one such regulator, Quaking 5 (Qki5), as an RNAbinding protein (RNABP) that is expressed in early embryonic neural stem cells and subsequently down-regulated during neurogenesis. mRNA sequencing analysis in neural stem cell culture indicates that Qki proteins play supporting roles in the neural stem cell transcriptome and various forms of mRNA processing that may result from regionally restricted expression and subcellular localization. Also, our in utero electroporation gain-of-function study suggests that the nuclear-type Qki isoform Qki5 supports the neural stem cell state. We next performed in vivo transcriptome-wide protein-RNA interaction mapping to search for direct targets of Qki5 and elucidate how Qki5 regulates neural stem cell function. Combined with our transcriptome analysis, this mapping analysis yielded a bona fide map of Qki5-RNA interaction at single-nucleotide resolution, the identification of 892 Qki5 direct target genes, and an accurate Qki5-dependent alternative splicing rule in the developing brain. Last, our target gene list provides the first compelling evidence that Qki5 is associated with specific biological events; namely, cell-cell adhesion. This prediction was confirmed by histological analysis of mice in which Qki proteins were genetically ablated, which revealed disruption of the apical surface of the lateral wall in the developing brain. These data collectively indicate that Qki5 regulates communication between neural stem cells by mediating numerous RNA processing events and suggest new links between splicing regulation and neural stem cell states.

[Keywords: RNA-binding protein; HITS-CLIP; alternative splicing; quaking; neural stem cell; cell adhesion]

Supplemental material is available for this article.

Received May 1, 2017; revised version accepted September 14, 2017.

A given cell's transcriptomic output is enabled by various regulators that mediate transcription and post-transcriptional modifications. Ubiquitously expressed transcripts encoding abundant proteins (e.g., housekeeping proteins) are essential for basic cellular functions, while cell typespecific transcripts determine the characteristics of specific cell lineages as well as cell morphology, functions, and behavior. Regulation of the transcriptome is thought to be controlled by ubiquitous and/or cell type-specific DNA-binding transcription factors (Yamamizu et al.

\footnotetext{
${ }^{6}$ These authors contributed equally to this work.

Corresponding authors: myano@med.niigata-u.ac.jp, hidokano@a2.keio.jp Article published online ahead of print. Article and publication date are online at http://www.genesdev.org/cgi/doi/10.1101/gad.300822.117.
}

2013). In addition, it has become increasingly clear that RNA-binding proteins (RNABPs), which are major contributors to post-transcriptional regulation via their interaction with RNAs, also participate in the generation of cell type-specific transcriptomes (Darnell 2013; Gerstberger et al. 2014; Zhang et al. 2016).

Traditionally, biochemical analyses of RNABPs have consisted of in vitro reconstitution assays with cultured cells and/or recombinant proteins. Although such

(C) 2017 Hayakawa-Yano et al. This article is distributed exclusively by Cold Spring Harbor Laboratory Press for the first six months after the full-issue publication date (see http://genesdev.cshlp.org/site/misc/ terms.xhtml). After six months, it is available under a Creative Commons License (Attribution-NonCommercial 4.0 International), as described at http://creativecommons.org/licenses/by-nc/4.0/. 
analyses are powerful and reliable methods for confirming biochemical mechanisms, these experiments often do not accurately reflect the stoichiometries of in vivo proteinRNA interactions. Thus, it has been necessary to develop improved assays capable of taking "snapshots" of RNABP function in vivo. Recently developed advanced technologies, such as protein-RNA interaction techniques, RNA sequencing (RNA-seq), and ribosome profiling, have begun to elucidate the biochemical functions of RNABPs at the transcriptomic level (Blencowe 2006; Licatalosi and Darnell 2010; Ingolia 2014; Nussbacher et al. 2015; Yano et al. 2015; Van Nostrand et al. 2016). Such systemic analyses provide precise mechanistic insights into gene regulation. These strategies, in combination with genetic models, have elucidated the biological roles of neural RNABPs. For example, such a strategy was used in the discoveries that Nova2 regulates the radial migration of postmitotic neurons and that Ptbp2 regulates the interkinetic migration of neural progenitors in the developing cortex (Yano et al. 2010; Licatalosi et al. 2012). Furthermore, disabled-1 signaling was identified as a target pathway mediating the Nova2-dependent cortical neuron migration phenotype. These reports illustrate how the study of RNABP-mediated RNA regulation using effective genetic tools and multilayered transcriptome assays is essential for developing a better understanding of the physiological functions of RNABPs.

One recent study reported that $\sim 1542$ genes encode RNABPs and that the expression patterns of a majority of these showed a degree of tissue specificity that was lower than that for transcription factors (Gerstberger et al. 2014). This suggests that many RNABPs support RNA processes that govern basic cellular events rather than mediate tissue-specific transcriptome characteristics. However, numerous other reports have shown the involvement of tissue-specific RNABPs in neuronal developmental processes and diseases. Specifically, Nova2-, Ptbp1/2-, and Srrm4-dependent alternative splicing controls early cortical development, and Stau2 regulates asymmetric cortical neural stem cell divisions, as evidenced by genetic tools and/or in vivo manipulations (Kusek et al. 2012; Li et al. 2014; Vuong et al. 2016; Zhang et al. 2016)

The Quaking (Qki) proteins are a STAR (signal transduction and activation of RNA) family of K homology (KH) domain-containing RNABPs and are widely expressed across multiple embryonic and adult tissues. A spontaneous mouse mutant, quaking ${ }^{\text {viable }}\left(q k^{V}\right)$, exhibits a deletion of the proximal region of the $Q k$ gene on mouse chromosome 17 and has been used to define Qki functions (Ebersole et al. 1996). Genetic and biochemical studies have reported that Qki is involved in certain aspects of RNA metabolism, such as pre-mRNA splicing, mRNA stabilization, microRNA (miRNA) biogenesis, and circular RNA (circRNA) biogenesis (Wang et al. 2013; Zong et al. 2014; Conn et al. 2015). In the nervous system, Qki proteins are predominantly expressed in oligodendrocyte lineage cells (Hardy et al. 1996; Hardy 1998) and are key regulators of oligodendrocyte differentiation and myelination. These effects are mediated by RNA-level regulation of cell cycle- and myelin-related genes (Larocque et al. 2002, 2005; Wu et al. 2002; Darbelli et al. 2016). In addition, our immunohistochemical analysis in the present study and a previous report revealed unique cellular distributions of these proteins during brain development (Fig. 1; Hardy 1998). Specifically, Qki5 is expressed in early neural stem cells but not neuronal progenitors (Fig. 1; Hardy 1998). However, the transcriptome-wide RNA-binding sites and biologically relevant target RNAs of the three major alternatively spliced isoforms (Qki5, Qki6, and Qki7) in neural stem cells remain unclear. Our mRNA-seq analysis of $Q k$ knockdown neural stem cells presented here suggests that Qki5 facilitates the generation of a neural stem cell-specific transcriptome, a function consistent with the restricted expression pattern of these proteins. Additionally, transcriptomewide mapping of Qki5-RNA interactions via highthroughput sequencing and cross-linking immunoprecipitation (HITS-CLIP), a method used previously to define the functional roles of RNABPs, revealed that the major functions of Qki5 involve binding to intronic RNA and the regulation of alternative splicing in a binding position-dependent manner. Furthermore, we generated a list of direct Qki5 targets, suggesting previously unrecognized physiological roles for Qki5 in the regulation of cell adhesion pathways in neural stem cells. These functions were further confirmed by the use of newly generated conditional Qki knockout mouse models. Last, we illustrate how transcriptome-wide RNA methods and mouse genetics can be used to reveal a previously unsuspected Qki5 function. Specifically, we show that Qki5 regulates ventricular surface structure by mediating the cadherincatenin signaling pathway in early embryonic neural stem cells.

\section{Results}

Qki proteins are expressed in early neural stem cells

The Qki RNABPs are well-known regulators of the cell cycle and the differentiation of glial cells, such as oligodendrocytes and astrocytes, in the central nervous system (CNS) (Biedermann et al. 2010). Biochemical and mouse genetic studies have shown that Qki proteins regulate trasncriptomes via pre-mRNA splicing as well as mRNA stabilization and translation. However, comprehensive studies of the functions of Qki proteins in the developing CNS have not been reported previously. As each Qki protein isoform transcribed from the $Q k$ gene may differ in its localization and distribution in the embryonic brain, we first assessed Qki protein expression by immunofluorescence using isoform-specific antibodies (Supplemental Fig. S1). We found that Qki5 and Qki6 are expressed in the ventricular zone (VZ) at embryonic day 9.5 (E9.5), when neural tube closure has occurred, whereas expression of Qki7 begins at around E18.5, at the start of gliogenesis (Fig. 1A,D).

We next examined expression of Qki5 and Qki6 in neural stem cells using antibodies against various lineage markers in the E15.5 cortex. We observed that Qki5 and 

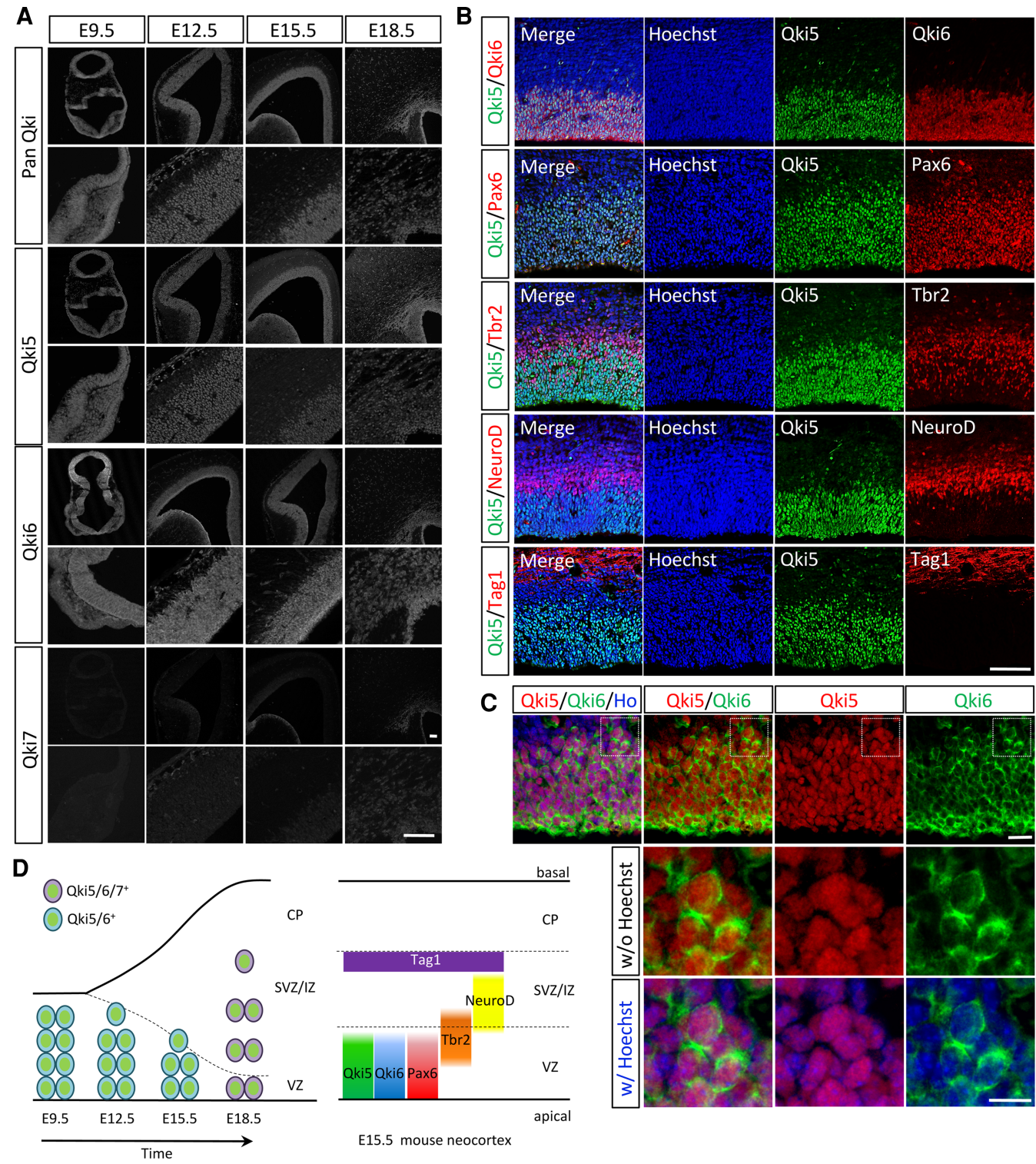

Figure 1. Qki5 and Qki6 are expressed in early neural stem cells of the developing mouse brain. $(A)$ Frontal sections of mouse brains at each developmental age (embryonic day 9.5 [E9.5], E12.5, E15.5, and E18.5) were immunostained using anti-pan-Qki antibodies or isoform-specific antibodies (Qki5, Qki6, and Qki7). This staining revealed that Qki5 and Qki6 are expressed in the ventricular zone (VZ) at E9.5, when neural tube closure is complete. Expression of these proteins continues through embryogenesis. Expression of Qki5 and Qki6 in neural stem cells is down-regulated during neurogenesis. Bar, $100 \mu \mathrm{m}$. (B) Detailed expression of Qki5 and lineage marker proteins in an E15.5 mouse cortex. Qki5-positive cells (green) also express Qki6 (red) in the VZ, although the subcellular localization of these proteins is quite different, with Qki5 being present in the nucleus, and Qki6 present in the cytoplasm. Qki5 expression colocalizes with Pax6 (red), a neural stem cell marker, and is down-regulated in Tbr2-positive intermediate progenitors (red) in the VZ and subventricular zone (SVZ). Qki5 and NeuroD (red) are mutually exclusive, and Qki5 expression is completely absent in Tag1-positive axons (red). Bar, $100 \mu \mathrm{m}$. $(C)$ Mutually exclusive subcellular localization of Qki5 and Qki6 proteins in embryonic neural stem cells from an E15.5 mouse neocortex. Qki5 (red) is clearly distributed in the nucleus stained with Hoechst 33258 (blue). In contrast, Qki6 (green) is localized mainly in the cytoplasm. The bottom two panels are higher-magnification views of the boxed area of the top panels. Bars: top panels, $20 \mu \mathrm{m}$; higher-magnification views, $10 \mu \mathrm{m} .(D)$ Schematic illustrations of the expression patterns of Qki proteins in the developing neocortex. (Left panel) Qki5 and Qki6 are expressed in embryonic neural stem cells at E9.5, and Qki7 expression starts at around E18.5. (Right panel) The expression of Qki proteins with lineage marker proteins in the E15.5 mouse neocortex. Qki5/6 expression is notably down-regulated in Tbr2+ intermediate progenitors. (CP) cortical plate; (IZ) intermediate zone.

Qki6 colocalize in the same cells of the VZ; however, their intracellular distributions differ. Qki5 is localized primarily in the nucleus due to its NLS (nuclear localization sig- nal) in the C-terminal region, while Qki6, which does not contain an NLS, is cytoplasmic (Fig. 1C). These two proteins are also coexpressed in Pax6-positive neural stem 
cells. Qki5 expression is down-regulated in cells that express Tbr2, which encodes the DNA-binding transcriptional factor Eomes (Englund et al. 2005). Tbr2 is also known as a marker of intermediate progenitors in the VZ and subventricular zone (SVZ) and is expressed mutually exclusive of the transcription factor NeuroD1, which is expressed in post-mitotic immature neurons (Fig. 1B,D; Miyoshi and Fishell 2012). Qki5 expression is furthermore absent in Tag1-positive axons. These expression patterns collectively suggest possible roles for Qki5 and Qki6 in neural stem cells during the early phase of embryonic neurogenesis.

\section{Qki is involved in neural stem cell differentiation through mediating RNA regulation}

To investigate whether Qki5 and Qki6 are involved in neural stem cell functions, we performed transcriptome analysis of mRNA obtained from cultured neural stem cells, which were characterized previously as expressing Nestin protein ( 95\% of cells), a marker of neural stem cells (Lendahl and McKay 1990; Hayashi et al. 2015). Neural stem cells were isolated from E14.5 mouse cortices and infected with a sh-Qk-or sh-control-expressing lentivirus to knock down all Qki proteins in cultures (Supplemental Fig. S2A-D). Six days after infection, the cells were collected and subjected to mRNA library generation and sequencing analysis. We confirmed the quality of shRNA against the $Q k$ gene by Western blotting analysis in HEK293T cells to detect the effect of exogenous Qki proteins (Supplemental Fig. S2C) and by immunofluorescence staining to detect endogenous Qki proteins in primary neural stem cells (Supplemental Fig. S2D).

We generated mRNA libraries from three independent neural stem cell cultures and performed 100-base-pair (bp) paired-end sequencing using the Illumina HiSeq 2500 system. In total, 366 million and 343 million paired-end reads were obtained for the control and shQk mRNA-seq samples, respectively. Sequence reads were aligned to the mouse genome ( $\mathrm{mm}$ ) $)$ or an exon junction database using the TopHat2 tool (Kim et al. 2013), and gene expression level and alternative splicing events were quantified using the Quantas tool (Wu et al. 2013). The analysis of gene expression changes confirmed a $>60 \%$ reduction in $Q k$ mRNAs. In shQk knockdown cells, steady-state levels of 202 transcripts were up-regulated (fold change $>1.5$; Stat_ebayes $<5 ; \quad P<0.001)$, and 483 transcripts were down-regulated (fold change $<0.66$; Stat_ebayes $<5 ; P<$ 0.001) with empirical Bayes statistics (Fig. 2A).

To explore how $Q k$ knockdown affects biological function in neural stem cells, we performed a gene ontology (GO) analysis using a list of genes that the mRNA-seq data indicated were up-regulated or down-regulated (Supplemental Fig. S3A). The following GO terms were ranked among the top five when genes that were up-regulated upon Qk knockdown were considered in biological processes: "brain development," "axonogenesis," "forebrain development," "neuron projection morphogenesis," and "cell morphogenesis involved in differentiation." These
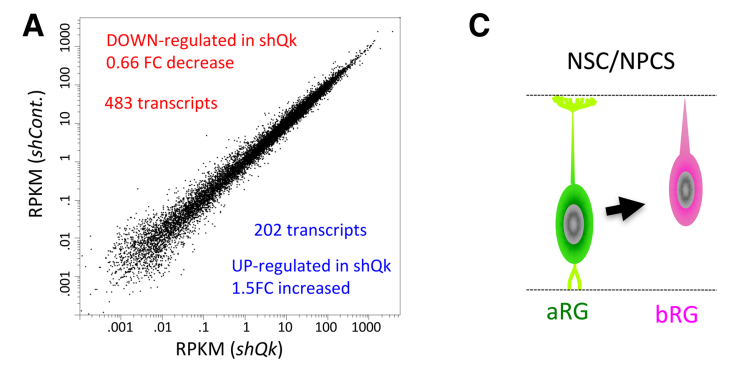

\section{B}

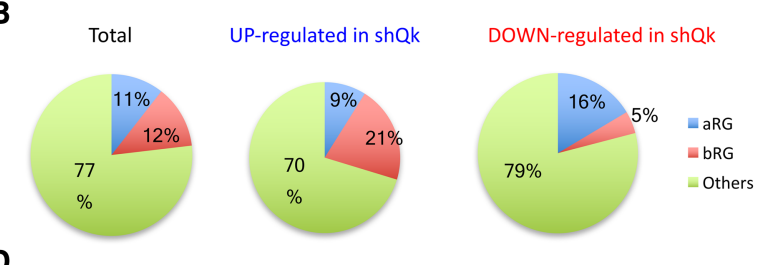

D

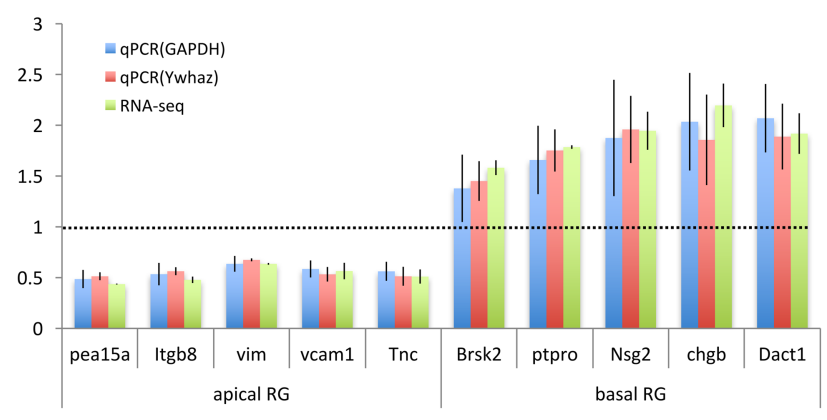

Figure 2. Qki5 and QKi6 are involved in neural stem cell function. Neural stem cells from the E14.5 mouse cortex were cultured and infected with a lentivirus encoding sh-control or shQk shRNA to knock down all Qki proteins. Six days after infection, the cells were collected. mRNA libraries were made and subjected to next-generation sequencing. $(A)$ Scatter plot of the $\log _{10}$ RPKM (reads per kilobase per million) of sh-control versus sh-Qk RNA-seq significant hits $(P<0.001)$ with absolute fold changes of $\geq 1.5$. Each point represents the mean RPKM obtained from three replicates for an individual gene. $(B)$ Pie charts show proportions of apical radial glia (aRG)-related transcripts and basal radial glia (bRG)-related transcripts among the total number of transcripts as well as up-regulated and down-regulated transcripts in sh-Qk neural stem cells. (C) Schematic illustration of aRG and bRG. In sh-Qk neural stem cells, bRG-related transcripts are more highly enriched compared with the sh-control condition. $(D)$ Bar graph of RNA-seq data and quantitative RTPCR (qRT-PCR) validation of Qki-dependent transcript level changes in aRG-related (pea15a, Itgb8, vim, vcam1, and Tnc [Tanascin C]) and bRG-related (Brsk2, ptpro, Nsg2, chgb, and Dact1) genes. Transcript levels of aRG-related transcripts are decreased, while transcript levels of bRG-related transcripts are increased in sh-Qk neural stem cells. The error bar shows the SD using three independent biological replicates.

terms suggest that neuronal differentiation processes are promoted by Qk knockdown. However, unexpected biological processes were ranked among the top five terms when down-regulated genes were considered (Supplemental Fig. S2E). 
We next classified the transcripts into four categories ("neuron," "astrocyte," "oligodendrocyte," and "others") using a transcriptome database for all three types of cells purified from the mouse brain (Cahoy et al. 2008). The data showed that the proportion of neuron-related genes was enriched among up-regulated transcripts in shQk neural stem cells, while the proportion of glia (astrocyte and oligodendrocyte)-related genes was enriched in down-regulated transcripts (Supplemental Fig. S2F), consistent with the GO data indicating that $Q k$ knockdown leads to neuronal differentiation. Importantly, these findings were validated by quantitative RT-PCR (qRT-PCR) analyses, confirming expression level changes in all 15 candidate genes tested. Five of the neuron-related transcripts were increased 1.3-fold to 5.7-fold, and 10 glial transcripts were decreased 0.3-fold to 0.8-fold (Supplemental Fig. S2G), indicating that Qki5 may facilitate gliogenesis by inhibiting neurogenesis.

This result prompted us to further examine the involvement of Qki proteins in the neural stem/progenitor state. Cortical neural stem cells include apical radial glia (aRG), which undergo mitosis at the ventricular side of the VZ, and basal radial glia (bRG), which lack ventricular contacts and undergo mitosis in the SVZ. One previous study reported that the transcriptome of bRG cells is more similar to that of intermediate neuronal progenitor cells than to aRGs in mice (Florio et al. 2015). Based on these aRG and bRG data sets and considering the total detected transcripts, $11 \%$ of transcripts were aRG-related, and $12 \%$ were bRG-related. Among the up-regulated transcripts in shQk cells, $21 \%$ were related to bRG cells, a proportion that revealed enrichment relative to the total detected transcripts. In contrast, aRG-related genes are more enriched among down-regulated transcripts in $s h-Q k$ neural stem cells (Fig. 2B,C).

These findings were also completely validated in qRTPCR assays of RNA isolated from three replicates each of sh-control and sh-Qk neural stem cells. These analyses confirmed expression level changes in 10 of 10 candidate genes tested. Expression levels of aRG-related genes (pea15a, itgb8, vim, vcam1, and Thc [Tanascin C]) decreased 0.5 -fold to 0.7 -fold, while those of bRG-related genes (brsks, ptpro, Nsg2, chgb, and Dact1) increased 1.4-fold to 2.1-fold in knockdown cells (Fig. 2D). These results suggest that Qki may be involved in the maintenance of aRG cells through regulation of the neural stem cell transcriptomic profile.

To further assess whether the knockdown of Qk affects pre-mRNA processing events in neural stem cells, we analyzed mRNA-seq data at the exon junction level. We observed dramatic mRNA processing defects, which may be due to changes in potential Qki-dependent RNA processing events. The decrease in Qki protein level induced the exclusion of 483 alternative exons and the inclusion of 597 alternative exons, with significant differences in splicing levels in Qk knockdown cells compared with control cells (Fig. 3A). These differences included all alternative exon changes (i.e., cassette exon, mutually exclusive exons, tandem cassette exons, alternative $5^{\prime}$ site, alternative $3^{\prime}$ site, and intron retention) (Table 1). Qk
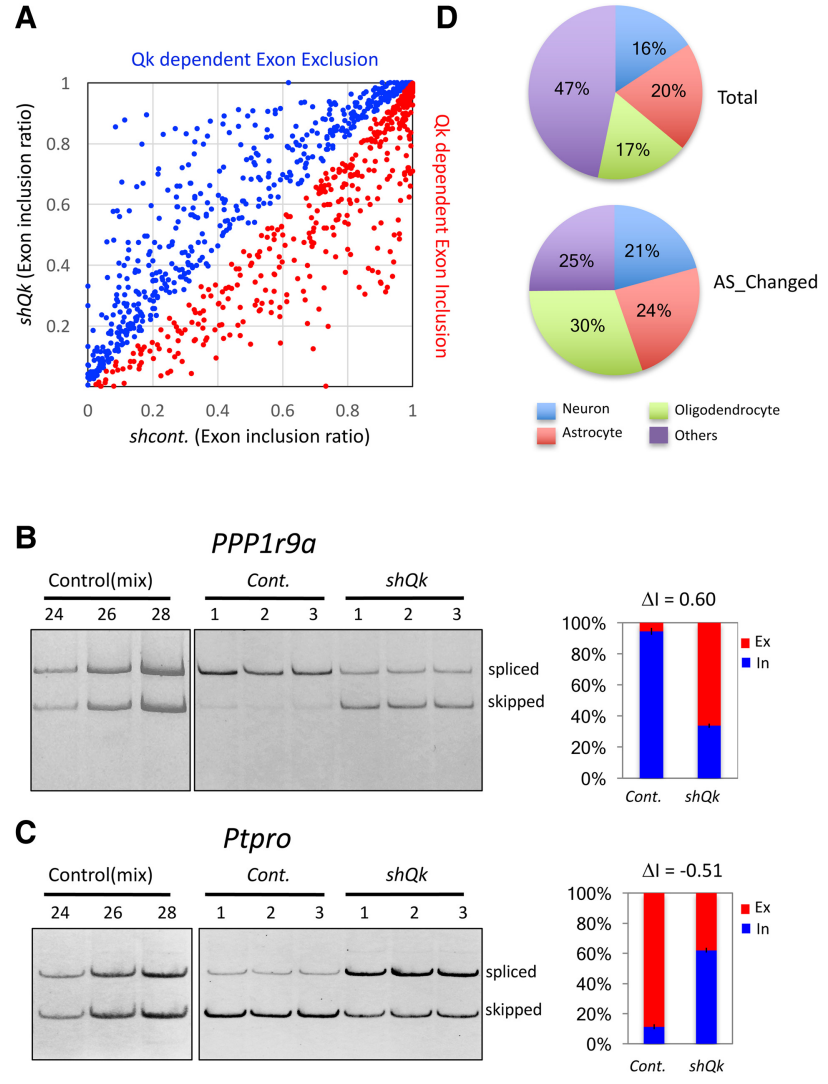

Figure 3. Qki-dependent alternative splicing in neural stem cells. (A) Scatter plot of the exon inclusion of sh-control versus sh-Qk RNA-seq significant hits. $P<0.001$. Each point represents the mean obtained from three replicates for an individual alternative splicing event. (B,C, left panels) RT-PCR analysis of $P P P 1 r 9 a$ $(B)$ and Ptpro $(C)$ splicing in both sh-Qk and sh-control neural stem cells. The control lanes correspond to cDNA mixtures amplified for different numbers of cycles (see the Materials and Methods). The bar graphs show the quantification of PPP1r9a $(B)$ and Ptpro $(C)$ RT-PCR products from the left panels. $\Delta \mathrm{I}$ indicates the change in fraction of the alternative exon. The error bar represents the SD using three independent biological replicates. $(D)$ Transcripts were categorized into four groups: "neuron," "oligodendrocyte," "astrocyte," and "others." The pie charts show the proportion of each group relative to all transcripts (top panel) and those transcripts with alternative splicing changes (bottom panel). Lineage genes were enriched in the Qki-dependent alternative splicing changes group.

knockdown primarily affected cassette-type exons 1618 of 1080 events; $57.2 \%$ ). In addition, semiquantitative RT-PCR validation was performed for two examples of cassette-type exons. Qki leads to the inclusion of $P P P 1 \mathrm{r} 9 a$ exon6 $(\Delta \mathrm{I}=0.60)$ and the exclusion of ptpro exon $17(\Delta \mathrm{I}=$ $-0.51)$, reflecting the mRNA-seq data (Fig. 3B,C). Transcripts likely to be regulated by Qki splicing changes were enriched in cell lineage-specific transcripts compared with total transcripts. Such transcripts included oligodendrocyte-related transcripts $30 \%$ of alternative splicing transcripts changed vs. $17 \%$ total), while no enrichment was observed for transcripts related to other 
Table 1. Qki5 regulates many types of alternative splicing patterns

\begin{tabular}{|c|c|c|c|}
\hline AS type & \# of events & $\%$ of events \\
\hline CE & 618 & 57.2 & \\
\hline TACE & 104 & 9.6 \\
\hline MXE & 51 & 4.7 \\
\hline A5SS & 85 & 7.9 & \hline \\
\hline A3SS & 151 & 14.0 \\
\hline IR & 71 & 6.6 & \hline \\
\hline
\end{tabular}

(CE) Cassette exon; (TACE) tandem cassette exon; (MXE) mutually exclusive exon; (A5SS) alternative $5^{\prime}$ site; (A3SS) alternative $3^{\prime}$ site; (IR) intron retention.

cell types, including neurons, astrocytes, and aRG and bRG cells (Fig. 3D; Supplemental Fig. S3A). In contrast, general transcripts that are expressed in multiple cell types were not enriched (Fig. 3D). Interestingly, the GO terms among splicing changes show that genes related to cellular organization, including cellular junctions, are enriched compared with those of transcript level changes (Supplemental Fig. S3B). The mRNA-seq data indicate that Qki proteins regulate RNA processing events that occur in the nucleus, to which the Qki5 isoform is restricted. We therefore suspected that Qki5 may be an important contributor to RNA processing events in neural stem cell functions.

\section{Qki5 is involved in neural stem cell functions}

To determine whether the nuclear isoform Qki5 functions in embryonic neural stem cells, we performed in utero electroporation experiments. These gain-of function experiments were performed to clarify the actions of Qki5 and Qki6, which are expressed in neural stem cells (Fig. 1). We introduced a plasmid encoding Flag-tagged Qki5 and Qki6 under control of the CAG promoter into the lateral ventricle (LV) of the developing neocortices of wild-type mice at E13.5. A control pCAGS empty vector or Qki5 or Qki6 pCAGS vector was transfected together with a plasmid encoding the fluorescent protein Venus (Nagai et al. 2002), enabling the monitoring of the transfected cells in vivo. Two days after the E13.5 electroporation, the brains were fixed for histological analysis via the assessment of Venus-labeled cells. In the control condition, Venus-labeled cells were widely distributed from the VZ to cortical plates. Transfection of plasmid encoding Qki5 protein caused cells to migrate to aberrant positions rather than that of Qki6 overexpression. We also observed a dramatic increase in the number of cells that were positive for phospho-histone $\mathrm{H} 3$ (PH3), a mitosis marker, in Qki5-overexpressing cells compared with control and Qki6-overexpressing cells (Fig. 4A,B). These results suggest that Qki5 inhibits neuronal migration and induces ectopic mitotic cells, supporting our hypothesis that the nuclear Qki member Qki5 plays functional roles in neural stem cells.

\section{Mapping of Qki5 protein and RNA interactions in the} developing mouse brain

mRNA-seq analysis identified Qki-regulated transcripts but did not address whether these targets are regulated directly or indirectly (Licatalosi and Darnell 2010). To efficiently identify direct Qki5 targets in living tissue, we performed BrdU (5-bromodeoxyuridine)-CLIP (WeynVanhentenryck et al. 2014) with several modifications (MY-HITS-CLIP; referred to here as HITS-CLIP) (M Yugami, H Okano, A Nakanishi, and M Yano, in prep.) using E14.5 mouse brains for material. After UV irradiation, Qki5-RNA complexes were seen in sizes similar to and higher-molecular-weight sizes than Qki5 in high and low levels of RNaseA, respectively (Fig. 5A). Purified RNA from triplicate individual brains were sequenced. The resulting reads (total 6,764,930 reads) were filtered and aligned to the mouse genome (Bowtie; mm9). After removing PCR duplicates, these unique tags were pooled
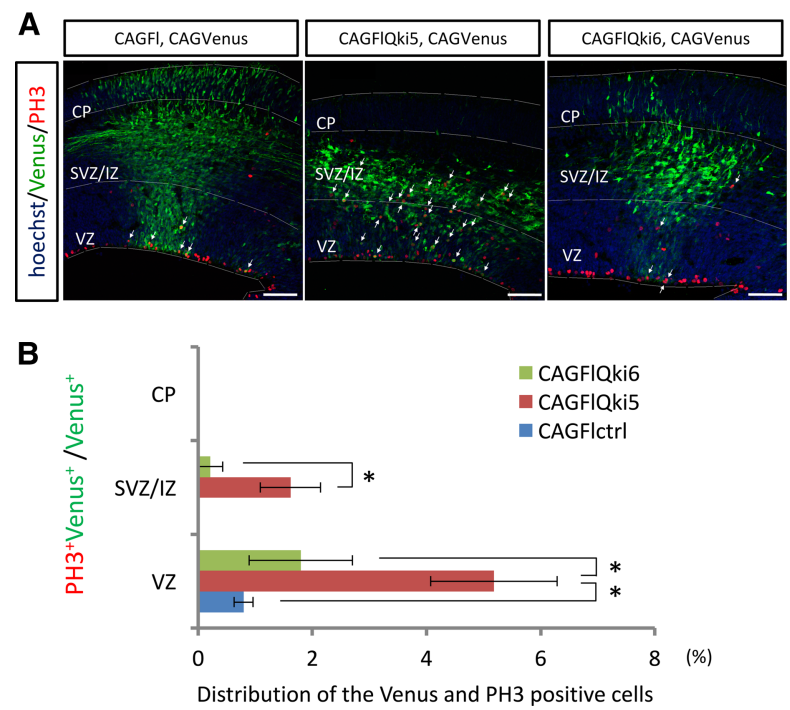

Figure 4. The nuclear Qki member Qki5 contributes to neural stem cells functions. (A) Gain-of-function study to clarify the actions of Qki5 and Qki6 (which are expressed in neural stem cells) by in utero electroporation. Plasmid DNA encoding Flag-tagged Qki5 and Qki6 under the control of the CAG promoter was introduced into the LV of the developing neocortices of wild-type mice at E13.5. To monitor the transfected cells, a plasmid encoding Venus protein was cotransfected. Two days after electroporation, the brains were fixed for immunofluorescence analyses. Qki5 transfected cells showed aberrant migration and positions of PH3-positive mitotic cells, whereas Qki6-overexpressing cells did not. (Blue) Hoechst; (red) PH3; (green) Venus. Bar, $100 \mu \mathrm{m}$. $(B)$ Quantification of $A$. The proportion of PH3/Venus double-positive cells relative to the total number of Venus-positive cells was significantly increased in the VZ and SVZ/IZ. $n=4 .\left(^{*}\right) P<0.05, t$ test. Data represent the mean SD of four independent brains. 

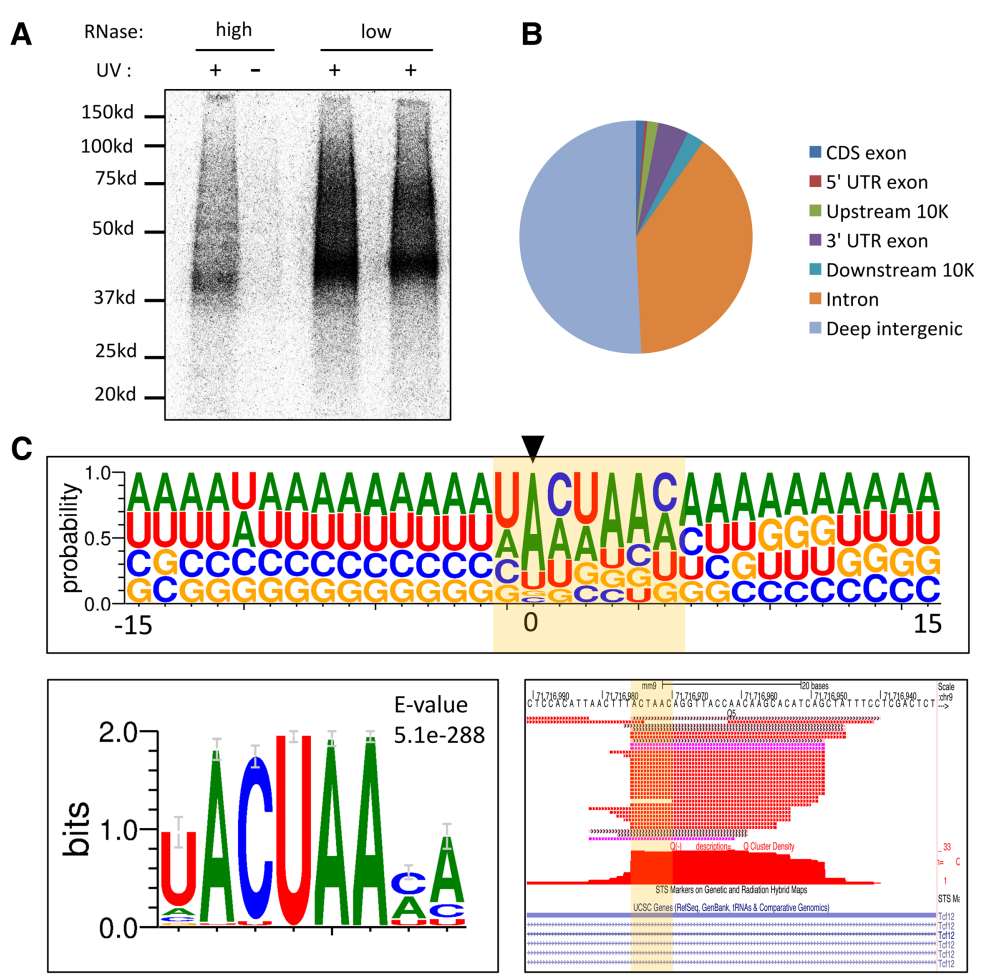

D

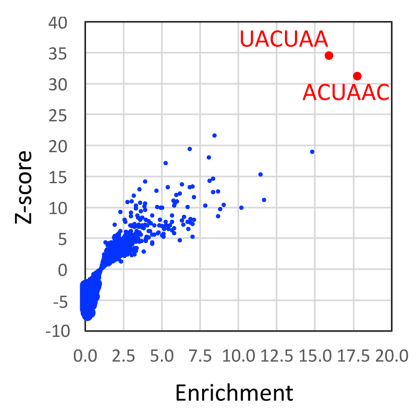

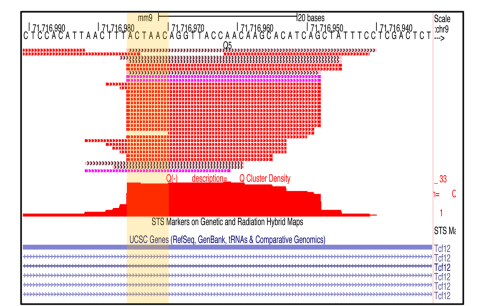

E

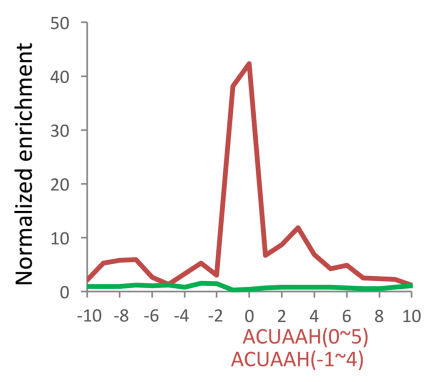

Figure 5. Qki5 HITS-CLIP identified specific binding sites in the mouse brain. (A) Autoradiogram of the nitrocellulose membrane of labeled ${ }^{32} \mathrm{P}$-labeled RNA crosslinked to immunoprecipitation-purified Qki5 from wild-type E14.5 mouse brains treated with low or high RNase A using an anti-Qki5-specific antibody. A very weak signal was observed under non-cross-linked conditions. (B) Genomic distribution of $\mathrm{BC} \geq 2$ clusters (Qki5binding events). ( $C$, top panel) CITS-based identification of ACUAAH as a consensus binding site for Qki5. (Bottom left panel) MEME (multiple EM [expectation maximization] for motif elicitation) analysis $[-15,15]$ also detected the UACUAAC motif as a top hit $(E$-value $=$ $5.1 \times 10^{-288}$ ). (Bottom right panel) A representative genome browser view of Qki5 CLIP tag density along the intronic region of the Tcf12 gene. Note that each Qki5-binding tag lines up sharply with the ACUAAH site, indicating a CITS. (D) Based on sequencing features associated with Qki5-RNA interactions, the most overrepresented $6 \mathrm{nt}$ were UACUAA and ACUAAC. UACUAA and ACUAAC have high $z$-scores: 34.44 and 31.21, respectively. (E) Enrichment analysis was calculated from the frequency of ACUAAH (red) and YCAY (green) starting at each position relative to the inferred truncated cross-link sites using extended sequences obtained from -15 to +15 relative to Qki5 CITSs and shuffled 31-nt sequences with the same nucleotide composition (controls). ACUAAH ( $\mathrm{H}$ indicates "not G ") were enriched at positions 0 and -1 .

to identify the Qki5-RNA interactions that were biologically reproducible. These interactions represented 11,931 clusters (biologic complexity $[\mathrm{BC}] \geq 2$; peak height $>2$ ) that contained overlapping Qki5 CLIP tags from all three brains $(185,426$ brain 1 tags, 93,117 brain 2 tags, and 49,673 brain 3 tags). Of these robust clusters, $41.6 \%$ and $43.1 \%$ were distributed in introns and deep intergenic regions of the mouse genome, respectively, potentially reflecting the cellular distribution of Qki5 (Fig. 5B). The tag length was 24 nucleotides (nt) in our CLIP experiments-shorter than for standard HITS-CLIP. Many of the tags aligned and appeared to begin at a single position, suggesting that the residue of cross-linked RNA sites bound by Qki5 blocked cDNA synthesis from RNA tags during reverse transcription; this process is known as cross-link-induced termination (Fig. 5C right, bottom panel; Weyn-Vanhentenryck et al. 2014). We therefore performed cross-link-induced termination analysis based on the Qki5 CLIP tags (Fig. 5C top panel; Supplemental Fig. S4A). Several past reports have used similar strategies to study in vivo protein-RNA-binding sites at single-nucleotide resolution and across the transcriptome (Modic et al.
2013; Weyn-Vanhentenryck et al. 2014). To identify the features that characterize Qki5-binding RNA sites, we obtained extended sequences from -15 to +15 relative to Qki5 CITSs (cross-linked-induced termination sites) and, as a control, shuffled 31-nt sequences with the same nucleotide content as the hits (Supplemental Fig. S4B). Based on the sequencing features associated with Qki5-RNA interactions, we found that the most overrepresented 6-nt features were UACUAA and ACUAAC, both of which showed very high significance ( $z$-scores: 34.44 and 31.21, respectively) (Fig. 5D right panel; Supplemental Fig. S4C). In addition, the top five ranked 6-nt features commonly included the CUAA sequence (Supplemental Fig. S4C).

Overall, our HITS-CLIP analysis from living brain tissue revealed robust Qki5 association with UACUAAH elements (where $\mathrm{H}$ indicates "not $\mathrm{G}^{\prime}$ ). This result is similar to a previously defined RNA-binding motif for Qki proteins, with slight differences /Galarneau and Richard 2005; Hafner et al. 2010). Among these elements, ACUAAH sequences are enriched 42-fold and 38-fold at the 0 and -1 positions (relative to the CITS), respectively. 
In contrast, the YCAY motif of Nova proteins, which share a KH-type RNA-binding domain with Qki proteins, were not enriched (Fig. 5E). Moreover, MEME (multiple EM [expectation maximization] for motif elicitation) analysis (in the -15- to 15-nt range) also revealed the UACUAAC motif with very high significance $(E$-value $=$ $\left.5.1 \times 10^{-288}\right)$ (Fig. 5C left, bottom panel; http://meme.ebi. edu.au). This finding agrees well with the CITS result. In fact, a representative CITS-containing element (ACUAAC) in an intronic sequence of the Tcf12 gene was observed at the 0 position of the CITS (Fig. 5C).

HITS-CLIP results with transcriptome analysis reveal a functional role of Qki5-dependent alternative splicing in neural stem cells

We generated Qki5-RNA-binding and RNA expression maps for both control and $Q k$ knockdown neural stem cells (Figs. 2, 5). Both data sets have been used to correctly predict RNABP rules, specifically with regard to alternative RNA processing (Licatalosi and Darnell 2010). We performed validation analysis of the mRNA-seq results using semiquantitative RT-PCR assays to measure the levels of transcripts containing potential Qki5-dependent exon inclusion or skipping. These experiments strongly validated the sequencing results (Supplemental Fig. S5A-H). In addition, we focused on the map $4 k 4$ gene, which contains several Qki5-RNA-binding sites as determined by HITS-CLIP (Supplemental Fig. S5I). Two large Qki5-RNA-binding clusters were identified in this gene, both of which were located in intronic regions and included alternative exons. These two clusters contained ACUAAC and ACUAAU sites (as identified by HITSCLIP), respectively (Supplemental Fig. S5I).

To determine whether these Qki5-RNA interactions are functionally relevant with respect to alternative exon inclusion in map4k4, we performed RT-PCR assays. We detected aberrant splicing changes, including multiple exon inclusions in Qk knockdown cells, suggesting that Qki5 directly regulates alternative splicing and has a functional role at this RNA-binding locus (Supplemental Fig. S5I). To identify the functional effects of Qki5, using the RNA sequence elements that we identified via HITSCLIP, we plotted the frequency of the identified sequence element ACUAAY across 278 cassette-type alternative splicing events. This analysis indicated an asymmetric bidirectional pattern of alternative splicing events. Specifically, Qki5 binding at this element on a $3^{\prime}$ end of an upstream intron results in exon skipping, whereas binding to the $5^{\prime}$ end of a downstream intron results in exon inclusion (Supplemental Fig. S5J).

To further explore the relationship between direct Qki5 binding and splicing, we examined HITS-CLIP-identified element clusters near alternative exons that were differentially expressed between control and $Q k$ knockdown neural stem cells, based on the mRNA-seq analysis. We restricted our analysis to exons that were subjected to cassette-type regulation. For the 296 Qki5-dependent cassette-type splicing events that were defined (with |DI| >
0.1 by mRNA-seq: 146 Qki5-dependent exon inclusion events and 150 Qki5-dependent skipping events), we performed normalized complexity mapping by counting the Qki5 CLIP tags within the region extending between the constitutive exons that flanked Qki5-sensitive exons. This method has been described previously in the generation of a Nova2 splicing map (Fig. 6A; Licatalosi et al. 2008). Our results suggest that the direction of Qki5-dependent alternative splicing can be predicted based on a position-dependent RNA map obtained using CLIP data from the developing mouse brain (Fig. 6A).

In addition, we confirmed three models of the Qki5binding position-dependent alternative splicing in neural stem cells using a series of representative validations, including Tcf12 (a neural stem cell transcription factor gene), in which Qki5-dependent exon skipping occurred; Evl, a gene encoding an actin-binding protein; and spag9, a gene encoding a member of the cancer testis antigen gene family in which Qki5-dependent exon inclusion occurred (Shankar et al. 1998; Uittenbogaard and Chiaramello 2002; Michael et al. 2010; Pollen et al. 2015). For these genes, Qki5-dependent exon inclusion and skipping were observed as well as both the Qki5-binding code and CLIP clusters close to the alternative exon along the present splicing rule (Fig. 6B-D).

\section{Qki5 regulates cell polarity of the apical surface by modulating the cadherin-catenin signaling pathway in neural stem cells}

Our finding that Qki5 mediates RNA processing raises the question of whether the Qki5 target sequence map and direct RNA targets are predictive of the biological significance of Qki5 in neural stem cells. To further explore the biologically relevant pathway of Qki5 targets in neural stem cells, we performed GO and KEGG (Kyoto Encyclopedia of Genes and Genomes) pathway analyses using a list of 892 predicted target genes identified by Qki5 HITS-CLIP (with CLIP cluster being defined as having more than five tags). "Adhesion," "junction," and "plasma membrane" were the recurring GO terms among Qki5 HITS-CLIP targets (Supplemental Tables S2-S4). KEGG pathways associated with Qki5 targets were cell adhesion molecules (CAMs) and tight junction proteins, some of which (cadherin and catenin) span these two groups and function in cell-cell adhesion, the cytoskeleton, etc. (Fig. 7; Supplemental Fig. S6; Supplemental Table S1).

These findings led us to investigate further whether Qki5 maintains cell-cell adhesion and the cytoskeleton in neural stem cells in vivo. As Qki-null mice die between E9.5 and E10.5 showing vascular remodeling defects in the yolk sacs and kinky neural tubes (Li et al. 2003), we generated a $Q k-f l o x$ mouse line. We then generated CNS-specific Qki conditional knockout (cKO) mice by crossing the Qk-flox mice with the Nestin-Cre driver line (Supplemental Fig. S7A,B; Tronche et al. 1999). The cadherin-catenin complex is highly concentrated at the apical surface of neural stem cells and participates in the organization of adherence junctions (AJs), as observed for epithelial cells (Hatta and Takeichi 1986; Aaku-Saraste 


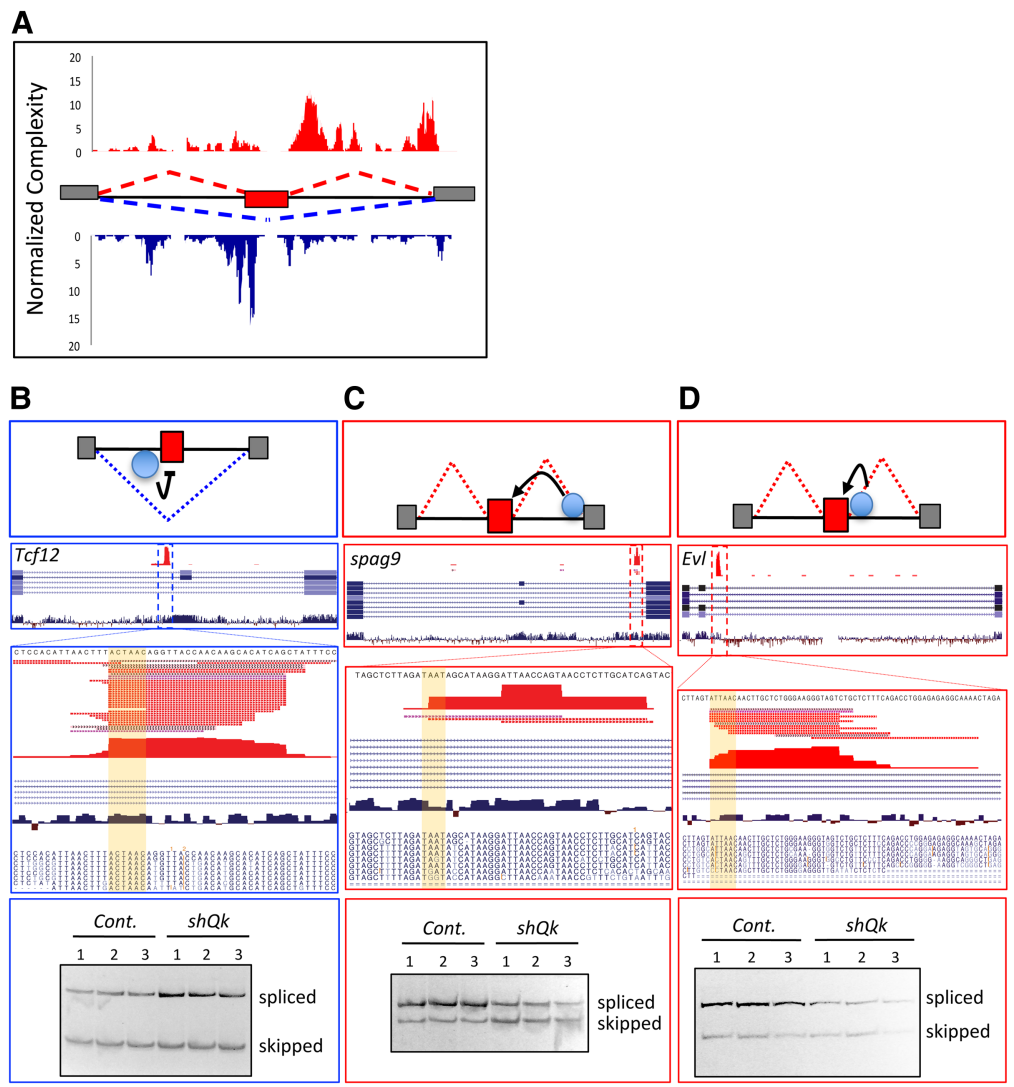

Figure 6. Qki-dependent bidirectional control of alternative splicing in vivo. (A) The normalized complexity map of CLIP tags near alternative exons that are misspliced in sh-Qk neural stem cells. The total number ( $Y$-axis) of CLIP tags centered in each 50-nt window relative to alternative and flanking constitutive splice sites is shown for exons that exhibit increased skipping (top panel) and splicing (bottom panel). (B-D, top panels) Three models of Qki5 action on exon inclusion and exclusion. (Second panels from the top) Genome browser view of Qki5 CLIP tag density along the $T c f 12(B)$, spag9 $(C)$, and $E_{V} l(D)$ genes, including the alternative exon and the flanking region. (Middle panels) Magnified view of each gene showing the Qki5-repressed alternative exon and a $\mathrm{BC} 2$ or BC3 CLIP cluster, including our defined Qki5-binding sites (highlighted in yellow). RT-PCR analysis of each pre-mRNA splice in sh-control and sh-Qk cells, performed to monitor the splicing of an alternative exons. (Bottom panels) Primers corresponding to each flanking exon were used to determine the expression of aberrantly processed mRNAs in sh-Qk cells. et al. 1996; Kusek et al. 2012). The AJ protein $\beta$-catenin binds both cadherins and actin fibers. Consistent with these prior studies, our immunohistochemical analysis revealed a polarized localization of $\beta$-catenin at the apical cell membrane in the control cortex at E14.5 (Fig. 8A). In addition, another AJ protein, $\mathrm{N}$-cadherin, exhibited intense localized expression at the apical surface, where AJs are located in neural stem cells (Supplemental Fig. S7C; Kadowaki et al. 2007). In the Qk cKO brain, we found that $\beta$-catenin and $\mathrm{N}$-cadherin levels were significantly reduced, especially on the ventricular surface of the ventro-caudal portion of the LV. At higher magnification, we observed a mesh-like pattern of unpolarized $\beta$-catenin expression at the apical surface, where delaminated bulges often formed (Fig. 8A).

More recently, $\beta$-catenin was identified as a component of centrosomes required for centrosome maintenance in neuroepithelial cells (Huang et al. 2007; Chilov et al. 2011). To further investigate the impact of Qki5 on neural stem cells, we analyzed centrosomes and microtubules. In control brains, centrosomes and microtubules on the ventral surface of the $L V$, as detected by $\gamma$-tubulin and a-tubulin, respectively, were localized at the apical surface as expected. In Qki cKO brains, $\gamma$-tubulin staining was irregular and expanded into the VZ of the LV. In these cells, the polarized localization of $\alpha$-tubulin was lost (Fig. 8A; Supplemental Fig. S7D). During neurogenesis, centrosome and microtubule organization is implicated in interkinetic nuclear localization in neuroepithelial cells. In the control cortices at E14.5, the majority of M-phase cell nuclei was labeled with PH3 at the apical surface of the VZ, and S-phase nuclei detected using $30 \mathrm{~min}$ of BrdU pulse-labeling were observed in the VZ/SVZ. In the $\gamma$-tubulin-disrupted area of Qki cKO cortices, PH3positive $M$-phase cells were not anchored to the apical surface and penetrated into the VZ. Moreover, BrdU-labeled S-phase cells were dispersed in the VZ/SVZ and invaded the ventricular surface. In addition, whereas Pax6positive neural stem cells were restricted to the VZ in control mice, Pax6-positive neural stem cells were located diffusely in the VZ/SVZ of Qki cKO mice, with an accompanying loss of apical localization of $\beta$-catenin (Fig. 8B; Supplemental Fig. S7E). These scattered Pax6-positive neural stem cells are also expressing the intermediate filament protein Nestin, another neural stem cell marker (Supplemental Fig. S7F). Last, we asked whether Qki5 impacts neurogenesis. Dcx-positive neurons were mislocalized in the apical surface and VZ of Qki cKO mice, suggesting that ectopic neurogenesis occurs in the ventricular regions of disorganized neural stem cells (Fig. $8 \mathrm{C})$. These observations were replicated in three separate Qki cKO cortices.

Given that Qki5 regulates cell polarity of the apical surface in neural stem cells, we sought to determine whether Qki5 directly regulates key alternative splicing events related to cadherin-catenin pathways predicted by GO analysis. A recent study identified several genes involved in the stemness of human neocortical radial glia (neural stem cells in the outer SVZ) that play an important role in cortical expansion (Pollen et al. 2015). Of those genes, 


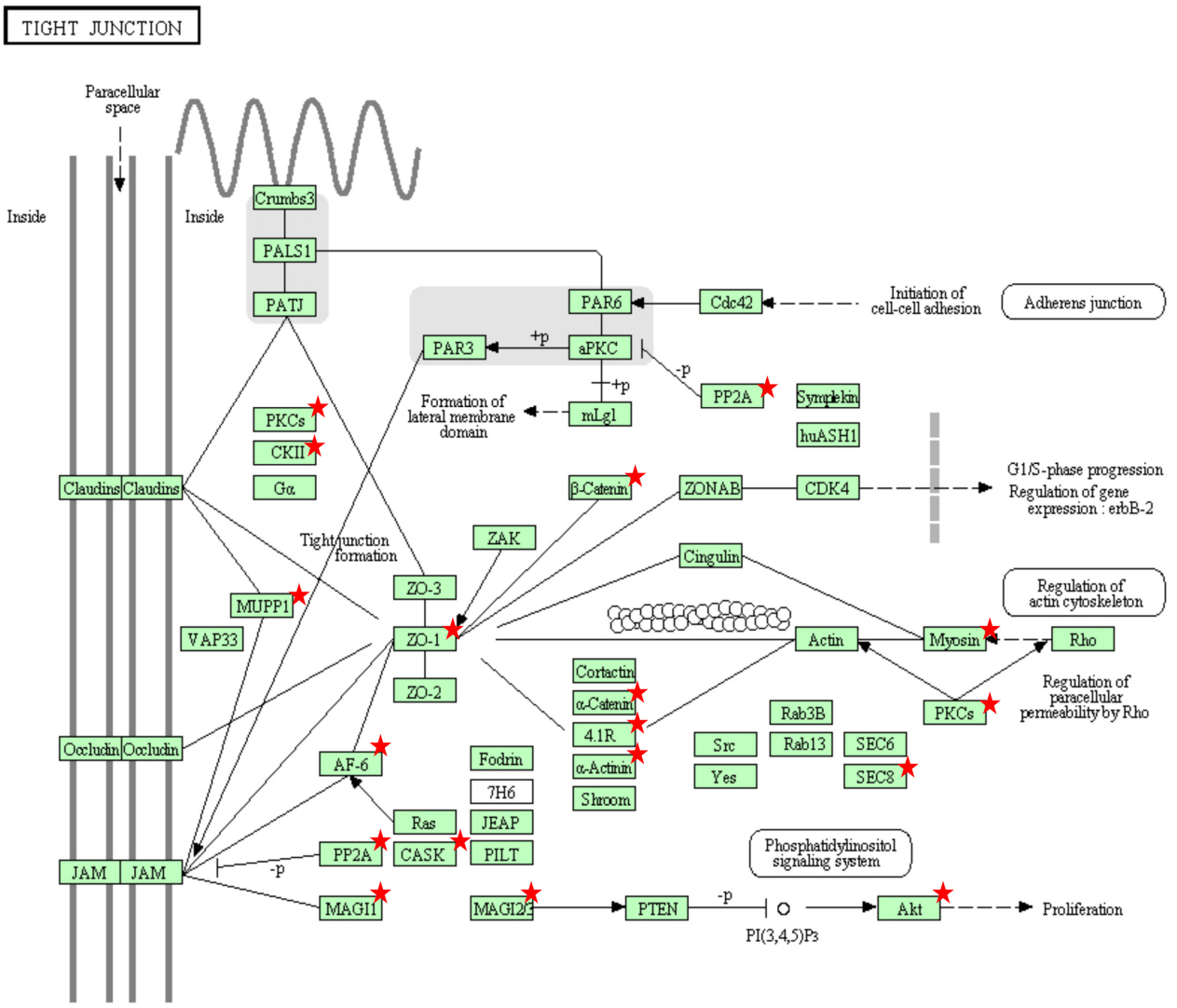

Figure 7. Tight junction pathway overrepresented among Qki5 HITS-CLIP target genes based on DAVID-KEGG pathway analysis. An example of a KEGG pathway that was overrepresented among the Qki5 targets. The red stars denote direct target genes identified by Qki5 HITS-CLIP (cut-off: CLIP cluster with more than five tags). The figures were obtained from GO analysis using DAVID Bioinformatics Resources (http://david.niaid.nih.gov).

The and Ptprz1 (protein tyrosine phosphatase, receptor type Z, polypeptide 1) each harbors Qki5-regulated alternative exons and is known to be involved in multiple neural stem cell functions such as epithelial-mesenchymal transition (EMT), cell adhesion, proliferation, and self-renewal. Both exons were validated by qRT-PCR and semiquantitative RT-PCR analysis using mRNA obtained from shQk knockdown cultured neural stem cells and primary cultured neural stem cells from E14.5 Qki cKO mouse cortices (Fig. 8D; Supplemental Fig. S8A). Furthermore, we discovered an important Qki5 direct RNA switch between pkm1 (pyruvate kinase, muscle 1) and $p k m 2$ as a potential major regulator of $\beta$-catenin signaling. RT-PCR analysis showed up-regulation of $P \mathrm{~km} 1 \mathrm{ex}-$ pression in both $s h Q k$ knockdown and Qki cKO neural stem cells (Fig. 8E; Supplemental Fig. S8B,C). Interestingly, this up-regulation of $P \mathrm{~km} 1$ was also observed in a $\mathrm{Qk}$ gene dosage-dependent manner using three different siRNAs for the Qk gene (Supplemental Fig. S8C), which may represent a mechanism similar to that observed for PTBP1 and hnRNPAl/2 (David et al. 2010). In addition, given that Qki5 regulates the cadherin-catenin pathway, we performed qRT-PCR analysis of mRNA obtained from primary cultured neural stem cells. We found the mRNA reduction of major $\beta$-catenin downstream genes, such as myc, ccnd1, $c d 44$, and vim (Fig. $8 \mathrm{~F}$ ). This primary Qki cKO neural stem cell culture model also confirmed our findings that Qki inhibits neurogenesis and maintains aRG-related genes (Fig. 2D; Supplemental Figs. S2G, S8D, E). Last, as Qki5 acts on the CAM pathway and the microenvironment of neural stem cells, these results are consistent with the KEGG pathway analysis of Qki5 HITS-CLIP direct targets.

Thus, the impaired polarization of cell polarity signals in early embryonic neural stem cells of Qki cKO brains may reflect a role for Qki5 in the developing brain (Fig. $8 \mathrm{G}, \mathrm{H})$. Failure of neural stem cells to maintain cell polarity results in disruption of the AJs, the detachment and displacement of neural stem cells from the VZ, and ectopic neurogenesis. These findings demonstrate that the regulation of RNA by Qki5 in neural stem cells is crucial for the maintenance of appropriate neural stem cell functions and neurogenesis.

\section{Discussion}

In the present study, we found that the RNABP Qki5 regulates neural stem cell characteristics and the cadherincatenin pathway through RNA processing. Through the 

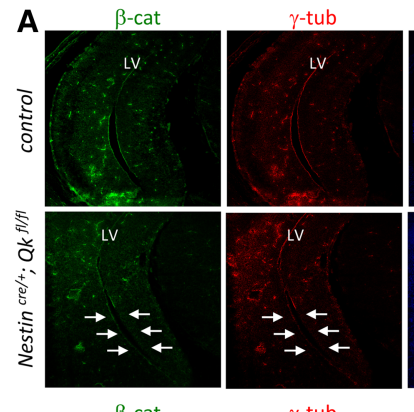

Hoechst

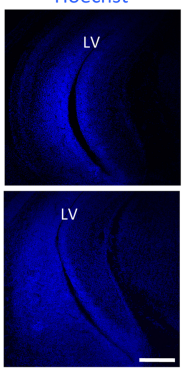

Hoechst
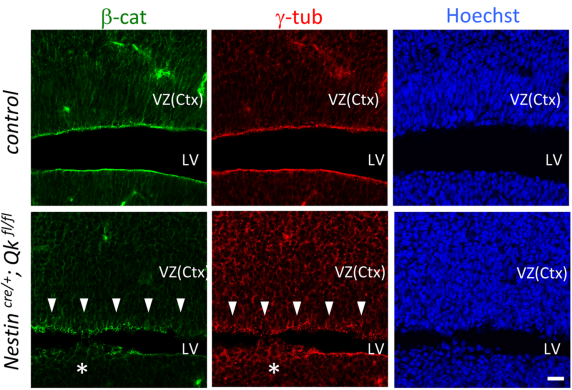
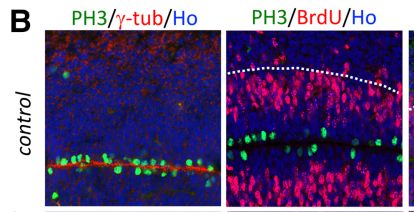

$\beta$-cat/Pax6/Ho

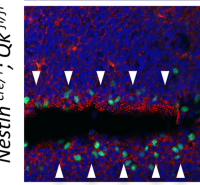

C $N-\operatorname{cad} / \mathrm{Dcx} / \mathrm{Ho}$
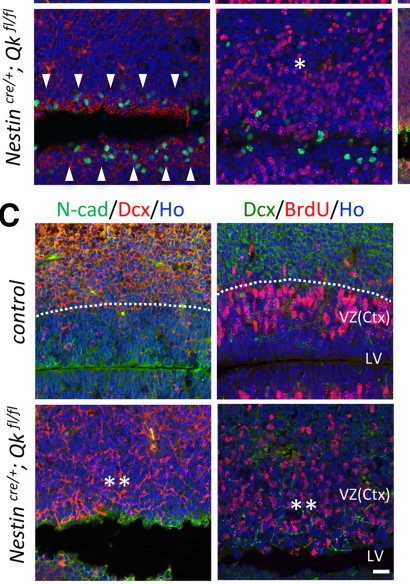

$\mathrm{Dcx} / \mathrm{BrdU} / \mathrm{Ho}$

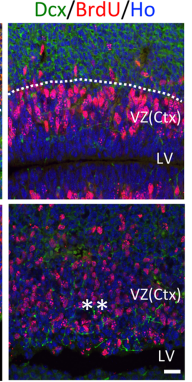

D
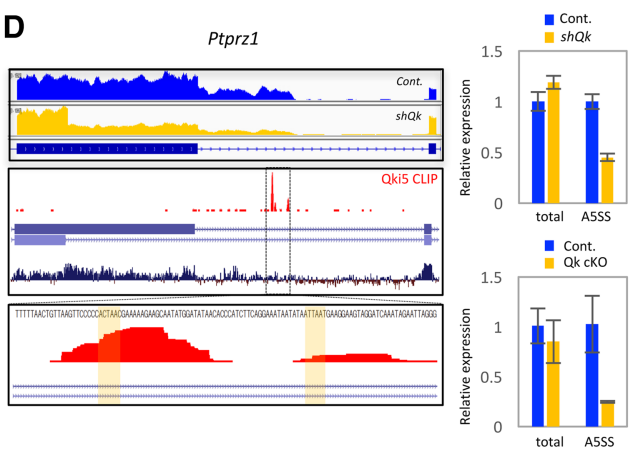

E

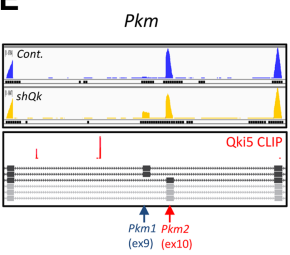

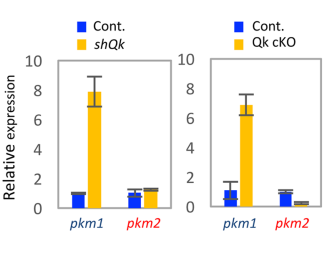

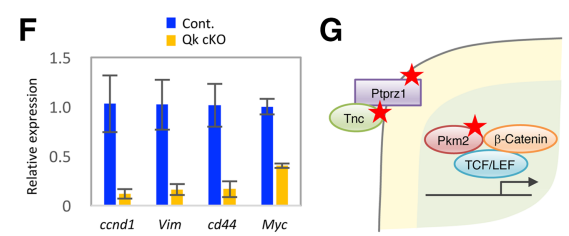

H

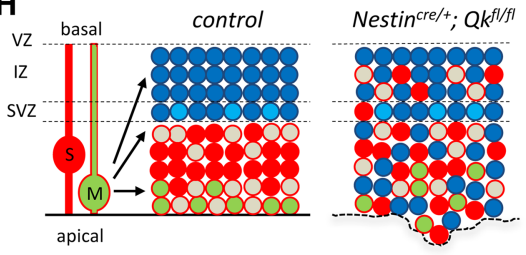

O Neuron $\left(\mathrm{DCX}^{+}\right)$O NSC $\left(\mathrm{Pax}^{+}, \mathrm{BrdU}^{+}\right)$
INP

O NSC $\left(\operatorname{Pax}^{+}\right.$

Figure 8. Qki deletion results in disruption of the apical surface of the lateral wall and ectopic neurogenesis. $(A-C)$ Double-immunostaining analysis of E14.5 control $\left(Q k^{f 1 / f 1}\right.$ or $\left.Q k^{f l /+}\right)$ and Nes-cre $/+; Q k^{f 1 / f 1}$ brains using antibodies against $\beta$-catenin and $\gamma$-tubulin $(A), \mathrm{PH} 3$ and $\gamma$-tubulin (B, left panel), $\mathrm{PH} 3$ and $\mathrm{BrdU}(B$, middle panel), $\beta$-catenin and Pax6 (B, right panel), N-cadherin and Dcx $(C$, left panel), and $\mathrm{Dcx}$ and $\mathrm{BrdU}(C$, right panel). Arrows indicate reduced protein expression, and arrowheads show defective areas. A single asterisk indicates a bulge-like structure in $A$ and expansion of BrdU-positive or Pax6-positive cells in $B$. Two asterisks indicate Dcx-positive neurons at the apical surface. Bars: $A$, top panel, $200 \mu \mathrm{m}$; $A$, bottom panel, $B, C, 20 \mu \mathrm{m}$. (D) Qki5 directs alternative splicing of the neural stem cell niche gene Ptprz1 (protein tyrosine phosphatase, receptor type Z, polypeptide 1). (Top left panel) Integrative Genomics Viewer (IGV) image showing the mRNA expression level along the Ptprz1 gene from sh-control versus sh-Qk neural stem cells. (Middle left panel) Genome browser view of Qki5 CLIP tag density along the Ptprz1 gene, including the alternative exon and the flanking region. (Bottom left panel) Magnified view of the Ptprz1 gene showing the Qki5-enhanced alternative exon and robust BC3 CLIP clusters, including defined Qki5binding sites (highlighted in yellow). (Right panel) qRT-PCR analysis in sh-control, sh-Qk, and Qk cKO neural stem cell cultures, performed to monitor the splicing of an alternative exon. ( $E$, top left panel) IGV image showing the mRNA expression level along the pkm (pyruvate kinase, muscle) gene from sh-control versus sh-Qk neural stem cells. Genome browser view of Qki5 CLIP tag density along the $\mathrm{pkm}$ gene, including the alternative exon and flanking region. (Bottom left panel) The intronic region of $p k m$ showing the Qki-inhibited alternative exon (pkm1) and a BC2 CLIP cluster centered on the 3 ' splice site. (Right panel) Bar graphs show the results of a qRT-PCR validation using sh-Qk and Qk cKO neural stem cells. (F) qRT-PCR analysis using cDNA from sh-control or sh-Qk neural stem cells. Four major $\beta$-catenin downstream genes exhibited decreased expression in $Q k \mathrm{cKO}$ neural stem cell culture. The data are represented as a ratio. The housekeeping gene Ywhaz was used as an internal control. (G) Qki5 contributes for the maintenance of neural stem cell functions by regulating alternative splicing of its key targets: Tnc, Ptprz1, and Pkm, as indicated by red stars. $(H)$ Model summarizing the roles of Qki proteins in neural stem cell organization and their functions in cortical development. The loss of Qki proteins leads to the ectopic placement of neural stem cells within the SVZ/IZ, with abnormal cell cycle-dependent interkinetic nuclear movement and ectopic neurogenesis. Qki may contribute to the polarized spatial distribution of crucial signaling complexes within neural stem cells and thus support neurogenesis and cortical development. 
use of systematic biological strategies, mRNA-seq, and HITS-CLIP analysis, we also revealed bidirectional regulation of Qki5-dependent alternative splicing with direct target RNAs in the developing brain. These data were also predictive of the biological functions of Qki5 in neural stem cells, and an additional newly generated genetic tool confirmed a role for Qki5 in cell adhesions. Our in vivo biochemical and biological analyses further enabled the investigation of correlations between observed molecular mechanisms and biological functions.

Given the evidence that RNABPs are broadly expressed across all tissues, in contrast to DNA-binding transcription factors, many RNABPs are thought to support basic cellular processes (Gerstberger et al. 2014). In this study, we found that Qki5 and Qki6 are specifically expressed in early neural stem cells during brain development (Fig. 1; Hardy 1998). Interestingly, the expression level of these proteins rapidly decreases in Tbr2-positive neuronal progenitors, indicating that they have cell type specificity in the developing brain despite their broad expression across tissues. This observation raised the possibility that Qki5 and Qki6 might generate a neural stem cell-specific transcriptome and that the loss of their expression could lead to neuronal commitment. Indeed, based on our mRNA-seq analysis, Qki proteins are involved in neural stem cell states and the inhibition of neuronal gene expression, supporting the hypothesis that the expression patterning of RNABPs reflects the transcriptome of the host cell (Fig. 2).

Several lines of evidence indicate that individual RNABPs participate in multiple layers of RNA regulation and thereby modify the transcriptome and translatome. In such a complex context, it is difficult to precisely describe the biological and cellular roles of RNABPs (Irimia and Blencowe 2012; Kapeli and Yeo 2012). While classical biochemisty has provided mechanistic insights into RNA regulation, there is a gap between what can be learned from biochemical experiments and the intricacies of biological processes. Recent studies using comprehensive biochemical approaches and genetics have attempted to bridge this gap (Darnell 2013). Such comprehensive analyses, in combination with genetic mouse models, provide an accurate picture of RNA processing mechanisms (Licatalosi and Darnell 2010). Using similar strategies, we successfully characterized the nuclear function of Qki5, with a focus on its alternative splicing mechanisms. These functions are very similar to those of Nova proteins, which were the first identified tissue-specific RNABPs. Specifically, it was found that when Qki5 binds RNA upstream of an intron, the alternative exon is skipped, whereas binding downstream from an intron enhances exon inclusion (Fig. 6; Ule et al. 2006; Licatalosi et al. 2008).

Several groups have shown that certain RNABPs control cortical development. Importantly, all of these results were validated in knockout mice or by using in vivo knockdown strategies (Kusek et al. 2012; Licatalosi et al. 2012; Shibasaki et al. 2013; Quesnel-Vallieres et al. 2015; Vuong et al. 2016). However, mice that are null for a given RNABP often exhibit pleiotropic phenotypes, which can make it difficult to explain a specific biological role through a single target RNA processing event. Nova proteins, for which it was shown that altered disabled-1 signaling and $\mathrm{Z}^{+}$agrin alternative splicing could be rescued by correcting disrupted RNA regulation events, provide two excellent examples of proteins for which comprehensive studies led to major advances (Ruggiu et al. 2009; Yano et al. 2010). In our study, using in vivo biochemical and comprehensive strategies, we identified a specific pathway that explained the observed phenotype; namely, cell adhesion formation defects on the embryonic ventricular surface (Fig. 8; Supplemental Fig. S7). These findings were achieved through the use of both comprehensive biochemical assays and genetics. In fact, while mRNA-seq data support a role for Qki proteins in neural stem cell differentiation, the data regarding direct Qki5 target genes are consistent with only two biological processes (Fig. 7; Supplemental Table S1; Supplemental Fig. S6). Both of these identified that a primary role of Qki5 is related to cell-cell contacts rather than proliferation and differentiation of neural stem cells. Especially, phenotypes observed in Qki cKO (namely, the disruption of AJs) were also seen in the loss of $\beta$-catenin and $\mathrm{N}$-cadherin function (Fig. 8A-C; Kadowaki et al. 2007; Chilov et al. 2011). Therefore, we conclude that this phenotype stems primarily from aberrant RNA regulation of Qki5 direct target genes and eventually results in mislocation of neural stem cells and ectopic neurogenesis. These processes highlight the importance of obtaining an accurate picture of the protein-RNA regulatory network.

One previous study revealed a role of Qki proteins as a tumor suppressor that is involved in p53 protein functions. This activity supported a model in which p53 directly up-regulates QKI protein, which mediates $m i R$ $20 a$ function and thereby inhibits TGF- $\beta$ signaling in GEM (glioblastoma multiforme) (Chen et al. 2012). In this previous study, QKI stabilized mature miR-20a via direct binding, without affecting the primary miRNA (primiRNA) levels of miR-20a. We did not observe any changes in miR-20a levels in Qki-depleted neural stem cells. Alternatively, another report has suggested a role for Qki proteins in miRNA processing and cancer. For example, Qki was reported to inhibit the expression of mir7-1, which is transcribed from intronic sequences of the hnrnpk gene. Qki proteins were observed to directly bind both upstream and downstream intronic RNA sites near the mir7-1 locus and affect cancer progression in a glioma cell line. This regulation is involved in tumor progression of the glioma cell line U343 (Wang et al. 2013). A similar form of regulation was also observed in the context of neural stem cells in the present study. Our CLIP data revealed two functional Qki5-binding elements in the upstream intron of mir7-1 but not in the downstream sites, and qRT-PCR validation assays confirmed Qki5-dependent mir-7-1 processing (Supplemental Fig. S8F). These results suggest that Qki5 proteins have context-dependent regulatory functions, emphasizing the significance of approaching these questions in a cellular context.

Genetic studies have implicated alternative splicing in a growing list of human diseases (Singh and Cooper 2012; Ling et al. 2013). For example, schizophrenia is 
strongly associated with the expression of aberrantly spliced isoforms of pathology-related genes, including Erbb4 and DISC1 (Law et al. 2007; Nakata et al. 2009). $\mathrm{Q} K I$ has also been reported as a candidate gene for schizophrenia (Aberg et al. 2006). A recent report showed that a long noncoding RNA (lncRNA), Gomafu, which is down-regulated in the post-mortem cortical gray matter of the superior temporal gyrus in schizophrenia, directly binds QKI proteins. These data suggest a model in which this interaction leads to aberrant splicing in schizophrenia pathology (Barry et al. 2014). Our CLIP data from neural stem cells also confirmed numerous Qki5-binding sites (consisting of a large number of ACUAAH sites) on gomafu transcripts (data not shown). However, we still do not have clear evidence of whether a Qki5-gomafu interaction affects Qki5-dependent alternative splicing events. As we were able to identify several Qki5 target transcripts in this study, further studies focusing on the potential interaction with gomafu are warranted, as gomafu knockout mice exhibited hyperactive behavior and a small subset of aberrant alternative splicing events in a recent report (Ip et al. 2016). In addition to RNA regulation, alternative mRNA splicing, miRNA processing, and lncRNA binding, Qki proteins are now known to be major regulators of circRNA generation. A previous finding estimated that QKI participates in over one-third of circRNA production during the human EMT (Conn et al. 2015). This observation of the role of Qki in the EMT may mirror our observations in the context of neural stem cells. Specifically, Qki-deficient mice exhibited defects in the neural stem cell microenvironment due to aberrant cell-to-cell contacts (Fig. 8; Supplemental Figs. S7, S8), similar to what occurs in the EMT. Therefore, the Qki5 regulatory network, including circRNA regulation, may also be involved in the EMT. Our findings can now be added to what is known regarding how RNABPs contribute to the numerous functions of RNA in cortical development. In addition, our data provide physiological insights into several fundamental processes, such as the roles of RNA in cell-to-cell communications.

Materials and methods

Mice

All of the mouse procedures were performed in accordance with Keio University and Niigata University guidelines. Conditional $Q k$-null mice $\left(Q k^{\text {flox/flox }}\right)$ were generated as follows. Briefly, a targeting construct containing loxP sites flanking exon 2 of the $q k$ gene was linearized and electroporated into C57BL/6N embryonic stem cells. Embryonic stem cell clones with homologous recombination at the targeting site were injected into ICR blastocysts. The chimeras obtained were subsequently crossed with C57BL/6N females, and offspring with successful germline transmission of the targeted allele were crossed with C57BL/6N mice expressing Flp recombinase to remove the neomycin resistance cassette. This breeding strategy resulted in a floxed allele $\left(Q^{f l o x /+}\right)($ Mishina and Sakimura 2007). The mice were then intercrossed to obtain $Q k^{\text {flox/flox }}$ homozygote animals.

Mouse tail DNA was genotyped by performing PCR with the specific primers Qk-loxF (5'-GACACCATCTTTACTTCCTG- $\left.3^{\prime}\right)$ and Qk-loxR (5'-TGTCAACCTATTCGGGCATT-3'), resulting in a band of $570 \mathrm{bp}$ in the wild-type and $680 \mathrm{bp}$ in the floxed allele. To generate embryonic conditional $Q k$ knockout mice, $Q k^{\text {flox/flox }}$ mice were crossed with Nestin-Cre transgenic mice (Tronche et al. 1999). To detect the Nestin transgenes and Cre recombinase, the following primers were used: Nes-F (5'-AA TCCTTCTTCGGGCTTCGG-3') and Cre-R (5'-TTGCGAACCT CATCACTCGT-3').

Neural stem cell culture and lentivirus production

Neural stem cells were isolated from the E14.5 mouse cortex and cultured in MHM (medium hormone mix) medium or KBM neural stem cell medium (Kojin Bio) with $10 \mathrm{ng} / \mathrm{mL}$ basic FGF (Hayashi et al. 2015). Lentiviruses encoding sh-control or sh-Qk, which targeted all Qki proteins for knockdown, were produced and infected into cultured neural stem cells following a previously described procedure (Naka et al. 2008). Six days after infection, the cells were collected and subjected to mRNA library generation and protein analysis.

\section{Tissue preparation and immunostaining}

The embryos were fixed with $4 \%$ paraformaldehyde in phosphate-buffered saline and post-fixed in the same fixative. The samples were then cryoprotected with $30 \%$ sucrose/PBS and sectioned (18- $\mu \mathrm{m}$ sections) with a cryostat (Leica, CM1850UV). For BrdU analysis, the dams were injected intraperitoneally with $\mathrm{BrdU}$ (Sigma-Aldrich) dissolved in $50 \mathrm{mg} / \mathrm{kg} 0.9 \% \mathrm{NaCl}$ for the indicated periods. The embryos were then subjected to immunohistochemistry. The sections were boiled in $10 \mathrm{mM}$ citric acid buffer ( $\mathrm{pH}$ 6.0) for $5 \mathrm{~min}$ before performing immunofluorescence staining for Qki5, Qki6, Qki7, PH3, BrdU, N-cadherin, $\beta$-catenin, $\gamma$-tubulin, $\beta$-tubulin, Nestin, Pax6, and Dcx. The sections were incubated overnight at $4^{\circ} \mathrm{C}$ with primary antibodies (see the Supplemental Material) followed by incubation with Alexa dye-conjugated secondary antibodies (1:1000; Invitrogen). The nuclei were stained with $10 \mu \mathrm{g} / \mathrm{mL}$ Hoechst 33258 (Sigma, B2883). Images of the immunostained specimens were collected using a confocal laser scanning microscope (Carl Zeiss, LSM510; or Olympus, FV1200).

\section{HITS-CLIP and data analysis}

CLIP using anti-Qki-5-specific antibodies (Bethyl Laboratories, A300-183A) was performed using E14.5 wild-type mouse whole brains with three biological replicates, as described elsewhere with several modifications (Licatalosi et al. 2008; Weyn-Vanhentenryck et al. 2014). Anti-rabbit IgG was used as a negative control. Following PCR amplification, high-throughput sequencing was performed using Illumina MiSeq at the Center for Bioresource, Brain Research Institute, Niigata University. The sequence tags were aligned to the mouse genome $(\mathrm{mm} 9)$ using Bowtie. Unique tags were collected by eliminating duplicates of those tags that had the same sequence start site on the same strand. Details are described in the Supplemental Material.

mRNA sequence and data analysis

Total RNA was obtained from three independent replicates of cultured neural stem cells. The RNA was extracted using QIAzol followed by the generation of mRNA libraries using Illumina TruSeq protocols for poly-A selection, fragmentation, and adaptor ligation according to the manufacturer's instructions (Illumina, TruSeq RNA sample preparation kit version 2). The multiplexed 
libraries were sequenced as 100-nt paired-end runs on an Illumina HiSeq 2500 system. Sequence reads were mapped to the reference mouse genome (NCBI build $37.1 / \mathrm{mm}$ ) ) by TopHat2 (Kim et al. 2013). Expression and alternative splicing events were quantified by Quantas tool (Wu et al. 2013). A detailed description is in the Supplemental Material.

\section{Western blot, RT-PCR, and $q R T-P C R$}

Western blotting and RT-PCR analyses were performed as described elsewhere (Yano et al. 2010). At least three biological replicates were used for qPCR, which was performed with a StepOnePlus real-time PCR detection system (Applied Biosystems). Mouse Ywhaz or Gapdh genes were used as internal controls, and the results were calculated with the $\Delta \Delta \mathrm{CT}$ method.

\section{In utero electroporation}

In utero electroporation was performed as described elsewhere (Yano et al. 2010). Briefly, empty pCAGS or Qki5- or Qki6expressing pCAGS vectors were transfected into E13.5 mouse brains together with a plasmid encoding Venus protein, which allows tracing of the transfected cells in vivo. Two days after electroporation, the brains were fixed and subjected to immunohistochemistry.

\section{Competing interest statement}

H.O. is a paid member of the Scientific Advisory Board of San Bio Co., Ltd., and K Pharma, Inc.

\section{Acknowledgments}

We are grateful to Dr. R.B. Darnell for encouragement, advice, and the use of the in-house bioinformatics tool, and Doug Sipp for proofreading. We also thank our colleagues, T. Otsuka, M. Horie, N. Bizen, M. Ito, and N. Yoshikawa for help and advice, and $M$. Hasegawa and S. Yamagiwa for technical assistance on experiments. This work was supported by grants from Shonan Incubation Laboratories (SIL) Research Fund from Takeda Pharmaceutical Company, Ltd. to M.Y. and H.O., and a Grant-in-Aid for Scientific Research on Innovative Areas (ncRNA neotaxonomy) from the Ministry of Education, Culture, Sports, Science, and Technology (MEXT) (grant no. JP17H05598), Grants-in-Aid for Scientific Research from the Japan Society for the Promotion of Science (JSPS) (grant nos. JP24700333 and JP16K07247), the Takeda Science Foundation for Medical Science Research, Keio Gijuku Academic Development Funds, the Mochida Memorial Foundation for Medical and Pharmaceutical Research, Basic Science Research Projects from the Sumitomo Foundation, GSK Japan Research Grant 2015, the Kanae Foundation, a research grant from the General Insurance Association of Japan, and the Initiative for the Implementation of the Diversity Research Environment (Niigata University) to M.Y. We are grateful to the sources of support to Y.H.-Y. (Grant-in-Aid for JSPS Research Fellow RPD, grant no. JP15J40150) and S.S. (Grant-in-Aid for JSPS Research Fellow DC1, grant no. JP10J05439).

\section{References}

Aaku-Saraste E, Hellwig A, Huttner WB. 1996. Loss of occludin and functional tight junctions, but not $\mathrm{ZO}-1$, during neural tube closure-remodeling of the neuroepithelium prior to neurogenesis. Dev Biol 180: 664-679.
Aberg K, Saetre P, Jareborg N, Jazin E. 2006. Human QKI, a potential regulator of mRNA expression of human oligodendrocyterelated genes involved in schizophrenia. Proc Natl Acad Sci 103: $7482-7487$.

Barry G, Briggs JA, Vanichkina DP, Poth EM, Beveridge NJ, Ratnu VS, Nayler SP, Nones K, Hu J, Bredy TW, et al. 2014. The long non-coding RNA Gomafu is acutely regulated in response to neuronal activation and involved in schizophrenia-associated alternative splicing. Mol Psychiatry 19: 486-494.

Biedermann B, Hotz HR, Ciosk R. 2010. The Quaking family of RNA-binding proteins: coordinators of the cell cycle and differentiation. Cell Cycle 9: 1929-1933.

Blencowe BJ. 2006. Alternative splicing: new insights from global analyses. Cell 126: 37-47.

Cahoy JD, Emery B, Kaushal A, Foo LC, Zamanian JL, Christopherson KS, Xing Y, Lubischer JL, Krieg PA, Krupenko SA, et al. 2008. A transcriptome database for astrocytes, neurons, and oligodendrocytes: a new resource for understanding brain development and function. J Neurosci 28: 264-278.

Chen AJ, Paik JH, Zhang H, Shukla SA, Mortensen R, Hu J, Ying H, Hu B, Hurt J, Farny N, et al. 2012. STAR RNA-binding protein Quaking suppresses cancer via stabilization of specific miRNA. Genes Dev 26: 1459-1472.

Chilov D, Sinjushina N, Rita H, Taketo MM, Mäkelä TP, Partanen J. 2011. Phosphorylated $\beta$-catenin localizes to centrosomes of neuronal progenitors and is required for cell polarity and neurogenesis in developing midbrain. Dev Biol 357: 259-268.

Conn SJ, Pillman KA, Toubia J, Conn VM, Salmanidis M, Phillips CA, Roslan S, Schreiber AW, Gregory PA, Goodall GJ. 2015. The RNA binding protein quaking regulates formation of circRNAs. Cell 160: 1125-1134.

Darbelli L, Vogel G, Almazan G, Richard S. 2016. Quaking regulates neurofascin 155 expression for myelin and axoglial junction maintenance. J Neurosci 36: 4106-4120.

Darnell RB. 2013. RNA protein interaction in neurons. Annu Rev Neurosci 36: 243-270.

David CJ, Chen M, Assanah M, Canoll P, Manley JL. 2010. HnRNP proteins controlled by c-Myc deregulate pyruvate kinase mRNA splicing in cancer. Nature 463: 364-368.

Ebersole TA, Chen Q, Justice MJ, Artzt K. 1996. The quaking gene product necessary in embryogenesis and myelination combines features of RNA binding and signal transduction proteins. Nat Genet 12: 260-265.

Englund C, Fink A, Lau C, Pham D, Daza RA, Bulfone A, Kowalczyk T, Hevner RF. 2005. Pax6, Tbr2, and Tbr1 are expressed sequentially by radial glia, intermediate progenitor cells, and postmitotic neurons in developing neocortex. I Neurosci 25: 247-251.

Florio M, Albert M, Taverna E, Namba T, Brandl H, Lewitus E, Haffner C, Sykes A, Wong FK, Peters J, et al. 2015. Human-specific gene ARHGAP11B promotes basal progenitor amplification and neocortex expansion. Science 347: 1465-1470.

Galarneau A, Richard S. 2005. Target RNA motif and target mRNAs of the Quaking STAR protein. Nat Struct Mol Biol 12: 691-698.

Gerstberger S, Hafner M, Tuschl T. 2014. A census of human RNA-binding proteins. Nat Rev Genet 15: 829-845.

Hafner M, Landthaler M, Burger L, Khorshid M, Hausser J, Berninger $\mathrm{P}$, Rothballer A, Ascano MJ, Jungkamp AC, Munschauer M, et al. 2010. Transcriptome-wide identification of RNAbinding protein and microRNA target sites by PAR-CLIP. Cell 141: 129-141. 
Hardy RJ. 1998. QKI expression is regulated during neuron-glial cell fate decisions. J Neurosci Res 54: 46-57.

Hardy RJ, Loushin CL, Friedrich VL, Chen Q, Ebersole TA, Lazzarini RA, Artzt K. 1996. Neural cell type-specific expression of QKI proteins is altered in quakingviable mutant mice. I Neurosci 16: 7941-7949.

Hatta K, Takeichi M. 1986. Expression of N-cadherin adhesion molecules associated with early morphogenetic events in chick development. Nature 320: 447-449.

Hayashi S, Yano M, Igarashi M, Okano HJ, Okano H. 2015. Alternative role of $\mathrm{HuD}$ splicing variants in neuronal differentiation. J Neurosci Res 93: 399-409.

Huang P, Senga T, Hamaguchi M. 2007. A novel role of phospho$\beta$-catenin in microtubule regrowth at centrosome. Oncogene 26: 4357-4371.

Ingolia NT. 2014. Ribosome profiling: new views of translation, from single codons to genome scale. Nat Rev Genet 15: 205-213.

Ip JY, Sone M, Nashiki C, Pan Q, Kitaichi K, Yanaka K, Abe T, Takao K, Miyakawa T, Blencowe BJ, Nakagawa S. 2016. Gomafu lncRNA knockout mice exhibit mild hyperactivity with enhanced responsiveness to the psychostimulant methamphetamine. Sci Rep 6: 27204.

Irimia M, Blencowe BJ. 2012. Alternative splicing: decoding an expansive regulatory layer. Curr Opin Cell Biol 24: 323-332.

Kadowaki M, Nakamura S, Machon O, Krauss S, Radice GL, Takeichi M. 2007. N-cadherin mediates cortical organization in the mouse brain. Dev Biol 304: 22-33.

Kapeli K, Yeo GW. 2012. Genome-wide approaches to dissect the roles of RNA binding proteins in translational control: implications for neurological diseases. Front Neurosci 6: 144.

Kim D, Pertea G, Trapnell C, Pimentel H, Kelley R, Salzberg SL. 2013. TopHat2: accurate alignment of transcriptomes in the presence of insertions, deletions and gene fusions. Genome Biol 14: R36.

Kusek G, Campbell M, Doyle F, Tenenbaum SA, Kiebler M, Temple S. 2012. Asymmetric segregation of the double-stranded RNA binding protein Staufen2 during mammalian neural stem cell divisions promotes lineage progression. Cell Stem Cell 11: 505-516.

Larocque D, Pilotte J, Chen T, Cloutier F, Massie B, Pedraza L, Couture R, Lasko P, Almazan G, Richard S. 2002. Nuclear retention of MBP mRNAs in the quaking viable mice. Neuron 36: $815-829$.

Larocque D, Galarneau A, Liu HN, Scott M, Almazan G, Richard S. 2005. Protection of $\mathrm{p} 27$ (Kip1) mRNA by quaking RNA binding proteins promotes oligodendrocyte differentiation. Nat Neurosci 8: 27-33.

Law AJ, Kleinman JE, Weinberger DR, Weickert CS. 2007. Disease-associated intronic variants in the ErbB4 gene are related to altered ErbB4 splice-variant expression in the brain in schizophrenia. Hum Mol Genet 16: 129-141.

Lendahl U, McKay RD. 1990. The use of cell lines in neurobiology. Trends Neurosci 13: 132-137.

Li Z, Takakura N, Oike Y, Imanaka T, Araki K, Suda T, Kaname T, Kondo T, Abe K, Yamamura K. 2003. Defective smooth muscle development in $q k I$-deficient mice. Dev Growth Differ 45: 449-462.

Li Q, Zheng S, Han A, Lin CH, Stoilov P, Fu XD, Black DL. 2014. The splicing regulator PTBP2 controls a program of embryonic splicing required for neuronal maturation. Elife 3: e01201.

Licatalosi DD, Darnell RB. 2010. RNA processing and its regulation: global insights into biological networks. Nat Rev Genet 11: $75-87$.
Licatalosi DD, Mele A, Fak JJ, Ule J, Kayikci M, Chi SW, Clark TA, Schweitzer AC, Blume JE, Wang X, et al. 2008. HITSCLIP yields genome-wide insights into brain alternative RNA processing. Nature 456: 464-469.

Licatalosi DD, Yano M, Fak JJ, Mele A, Grabinski SE, Zhang C, Darnell RB. 2012. Ptbp2 represses adult-specific splicing to regulate the generation of neuronal precursors in the embryonic brain. Genes Dev 26: 1626-1642.

Ling SC, Polymenidou M, Cleveland DW. 2013. Converging mechanisms in ALS and FTD: disrupted RNA and protein homeostasis. Neuron 79: 416-438.

Michael M, Vehlow A, Navarro C, Krause M. 2010. c-Abl, Lamellipodin, and Ena/VASP proteins cooperate in dorsal ruffling of fibroblasts and axonal morphogenesis. Curr Biol 20: 783-791.

Mishina M, Sakimura K. 2007. Conditional gene targeting on the pure C57BL/6 genetic background. Neurosci Res 58: 105-112.

Miyoshi G, Fishell G. 2012. Dynamic FoxG1 expression coordinates the integration of multipolar pyramidal neuron precursors into the cortical plate. Neuron 74: 1045-1058.

Modic M, Ule J, Sibley CR. 2013. CLIPing the brain: studies of protein-RNA interactions important for neurodegenerative disorders. Mol Cell Neurosci 56: 429-435.

Nagai T, Ibata K, Park ES, Kubota M, Mikoshiba K, Miyawaki A. 2002. A variant of yellow fluorescent protein with fast and efficient maturation for cell-biological applications. Nat Biotechnol 20: 87-90.

Naka H, Nakamura S, Shimazaki T, Okano H. 2008. Requirement for COUP-TFI and II in the temporal specification of neural stem cells in CNS development. Nat Neurosci 11: 1014-1023.

Nakata K, Lipska BK, Hyde TM, Ye T, Newburn EN, Morita Y, Vakkalanka R, Barenboim M, Sei Y, Weinberger DR, et al. 2009. DISC1 splice variants are upregulated in schizophrenia and associated with risk polymorphisms. Proc Natl Acad Sci 106: 15873-15878.

Nussbacher JK, Batra R, Lagier-Tourenne C, Yeo GW. 2015. RNAbinding proteins in neurodegeneration: seq and you shall receive. Trends Neurosci 38: 226-236.

Pollen AA, Nowakowski TJ, Chen J, Retallack H, Sandoval-Espinosa C, Nicholas CR, Shuga J, Liu SJ, Oldham MC, Diaz A, et al. 2015. Molecular identity of human outer radial glia during cortical development. Cell 163: 55-67.

Quesnel-Vallieres M, Irimia M, Cordes SP, Blencowe BJ. 2015. Essential roles for the splicing regulator nSR100/SRRM4 during nervous system development. Genes Dev 29: 746-759.

Ruggiu M, Herbst R, Kim N, Jevsek M, Fak JJ, Mann MA, Fischbach G, Burden SJ, Darnell RB. 2009. Rescuing $Z^{+}$agrin splicing in Nova null mice restores synapse formation and unmasks a physiologic defect in motor neuron firing. Proc Natl Acad Sci 106: 3513-3518.

Shankar S, Mohapatra B, Suri A. 1998. Cloning of a novel human testis mRNA specifically expressed in testicular haploid germ cells, having unique palindromic sequences and encoding a leucine zipper dimerization motif. Biochem Biophys Res Commun 243: 561-565.

Shibasaki T, Tokunaga A, Sakamoto R, Sagara H, Noguchi S, Sasaoka T, Yoshida N. 2013. PTB deficiency causes the loss of adherens junctions in the dorsal telencephalon and leads to lethal hydrocephalus. Cereb Cortex 23: 1824-1835.

Singh RK, Cooper TA. 2012. Pre-mRNA splicing in disease and therapeutics. Trends Mol Med 18: 472-482.

Tronche F, Kellendonk C, Kretz O, Gass P, Anlag K, Orban PC, Bock R, Klein R, Schütz G. 1999. Disruption of the glucocorticoid receptor gene in the nervous system results in reduced anxiety. Nat Genet 23: 99-103. 
Uittenbogaard M, Chiaramello A. 2002. Expression of the bHLH transcription factor Tcf12 (ME1) gene is linked to the expansion of precursor cell populations during neurogenesis. Brain Res Gene Expr Patterns 1: 115-121.

Ule J, Stefani G, Mele A, Ruggiu M, Wang X, Taneri B, Gaasterland T, Blencowe BJ, Darnell RB. 2006. An RNA map predicting Nova-dependent splicing regulation. Nature 444: 580-586.

Van Nostrand EL, Pratt GA, Shishkin AA, Gelboin-Burkhart C, Fang MY, Sundararaman B, Blue SM, Nguyen TB, Surka C, Elkins $\mathrm{K}$, et al. 2016. Robust transcriptome-wide discovery of RNA-binding protein binding sites with enhanced CLIP (eCLIP). Nat Methods 13: 508-514.

Vuong CK, Black DL, Zheng S. 2016. The neurogenetics of alternative splicing. Nat Rev Neurosci 17: 265-281.

Wang Y, Vogel G, Yu Z, Richard S. 2013. The QKI-5 and QKI-6 RNA binding proteins regulate the expression of microRNA 7 in glial cells. Mol Cell Biol 33: 1233-1243.

Weyn-Vanhentenryck SM, Mele A, Yan Q, Sun S, Farny N, Zhang Z, Xue C, Herre M, Silver PA, Zhang MQ, et al. 2014. HITSCLIP and integrative modeling define the Rbfox splicing-regulatory network linked to brain development and autism. Cell Rep 6: 1139-1152.
Wu II, Reed RB, Grabowski PJ, Artzt K. 2002. Function of quaking in myelination: regulation of alternative splicing. Proc Nat1 Acad Sci 99: 4233-4238.

Wu J, Anczuków O, Krainer AR, Zhang MQ, Zhang C. 2013. OLego: fast and sensitive mapping of spliced mRNA-Seq reads using small seeds. Nucleic Acids Res 41: 5149-5163.

Yamamizu K, Piao Y, Sharov AA, Zsiros V, Yu H, Nakazawa K, Schlessinger D, Ko MS. 2013. Identification of transcription factors for lineage-specific ESC differentiation. Stem Cell Reports 1: 545-559.

Yano M, Hayakawa-Yano Y, Mele A, Darnell RB. 2010. Nova2 regulates neuronal migration through an RNA switch in disabled-1 signaling. Neuron 66: 848-858.

Yano M, Ohtsuka T, Okano H. 2015. RNA-binding protein research with transcriptome-wide technologies in neural development. Cell Tissue Res 359: 135-144.

Zhang X, Chen MH, Wu X, Kodani A, Fan J, Doan R, Ozawa M, Ma J, Yoshida N, Reiter JF, et al. 2016. Cell-type-specific alternative splicing governs cell fate in the developing cerebral cortex. Cell 166: 1147-1162.e15.

Zong FY, Fu X, Wei WJ, Luo YG, Heiner M, Cao LJ, Fang Z, Fang $\mathrm{R}$, Lu D, Ji H, et al. 2014. The RNA-binding protein QKI suppresses cancer-associated aberrant splicing. PLoS Genet 10: e1004289. 


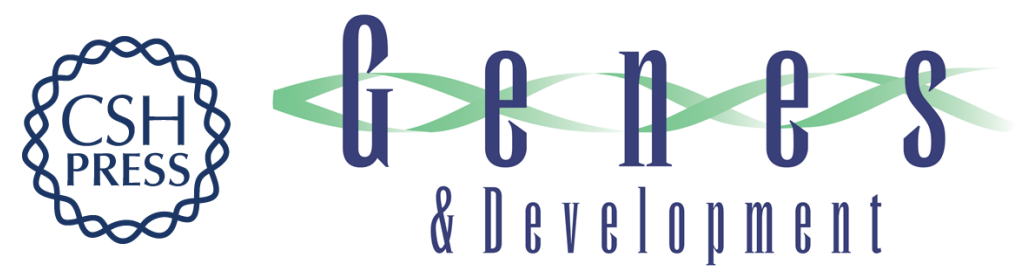

\section{An RNA-binding protein, Qki5, regulates embryonic neural stem cells through pre-mRNA processing in cell adhesion signaling}

Yoshika Hayakawa-Yano, Satoshi Suyama, Masahiro Nogami, et al.

Genes Dev. 2017, 31: originally published online October 11, 2017

Access the most recent version at doi:10.1101/gad.300822.117

\section{Supplemental http://genesdev.cshlp.org/content/suppl/2017/10/11/gad.300822.117.DC1 Material}

References This article cites 68 articles, 13 of which can be accessed free at: http://genesdev.cshlp.org/content/31/18/1910.full.html\#ref-list-1

Creative This article is distributed exclusively by Cold Spring Harbor Laboratory Press for the first Commons six months after the full-issue publication date (see

License http://genesdev.cshlp.org/site/misc/terms.xhtml). After six months, it is available under a Creative Commons License (Attribution-NonCommercial 4.0 International), as described at http://creativecommons.org/licenses/by-nc/4.0/.

Email Alerting Receive free email alerts when new articles cite this article - sign up in the box at the top Service right corner of the article or click here.

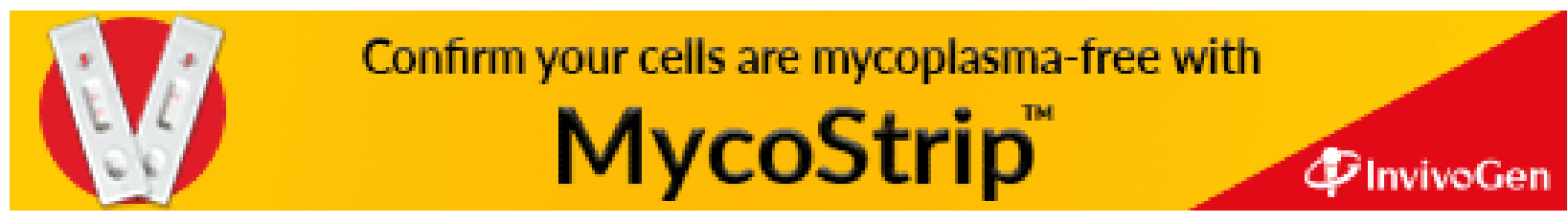

